\title{
UN ENFOQUE ALTERNATIVO PARA LA EVALUACIÓN Y DISEÑO DE EDIFICACIONES DE MAMPOSTERÍA EN ZONAS SÍSMICAS
}

\author{
Mario E. Rodríguez ${ }^{(1)}$
}

\begin{abstract}
RESUMEN
En este trabajo se evalúa la demanda y capacidad de deformación de edificaciones de mampostería dentro de un enfoque alternativo de evaluación y diseño por sismo de este tipo de edificaciones. Para esta evaluación se emplean registros de aceleraciones obtenidos en eventos sísmicos en los que se ha observado daños o colapsos en edificaciones de mampostería. También se emplea una medida de daño por sismo para la evaluación del comportamiento global de edificaciones de mampostería de tabique confinado. Los resultados de emplear esta medida se comparan con el comportamiento observado de este tipo de edificaciones en los eventos sísmicos estudiados. Estos resultados son congruentes con este comportamiento observado.
\end{abstract}

\begin{abstract}
Using an alternative approach, based on the demand and capacity of lateral deformations in the structure, an evaluation of seismic demand and capacity of masonry dwellings in seismic zones is performed in this paper. Recorded ground motions and general observed damage on masonry construction during several earthquakes that occurred mainly in Latin America are used in this evaluation. A seismic damage parameter previously proposed by the author is also used in a global evaluation of masonry dwelling responding to the analyzed earthquakes, in which the observed building response is correlated with the results of the damage parameter evaluation. Results of this correlation are considered in acceptable agreement.
\end{abstract}

\section{INTRODUCCIÓN}

La mampostería es un material ampliamente utilizado para la construcción de vivienda en el mundo y en particular en Latinoamérica, incluyendo México. El estudio del comportamiento estructural de edificaciones de mampostería es relevante en nuestro país por que la demanda de vivienda excede por mucho a la oferta disponible, y es deseable optimizar el empleo de materiales de manera de lograr una seguridad estructural aceptable.

En México en particular es importante conocer el comportamiento estructural de edificaciones de mampostería en zonas sísmicas, las que abarcan una gran parte del país (Manual

Artículo recibido el 13 de junio de 2002 y aprobado para su publicación el 23 de octubre de 2003. Se aceptarán comentarios y/o discusiones hasta cinco meses después de su publicación.

(1) Instituto de Ingeniería, UNAM, Ciudad Universitaria, CP 04510 México, DF. mrod@servidor.unam.mx, 
CFE93, Comisión Federal de Electricidad, 1993). Este tipo de edificaciones ha sido estudiado de manera extensa en México (Meli y Salgado, 1969; Meli, 1975; Alcocer et al., 1994), en Latinoamérica (Gallegos, 1989; García y Yamin, 1994; San Bartolomé, 2001), é incluso en diversos otros países del mundo (Maguenes y Calvi, 1997; Tomazevic y Klemenc, 1997). Una revisión de estos estudios indica que en éstos se ha hecho mayor énfasis en la evaluación de la capacidad resistente del material o de la estructura. Además, en años anteriores se contaba con poca información de las características de los movimientos sísmicos que podían afectar a la respuesta de estructuras. Una comparación del banco de datos de evaluación de daños en estructuras durante sismos y de registros de movimientos sísmicos existente antes de los años 90, indica que esta información de datos es bastante menor que la acumulada en los últimos años, por lo que es de interés el empleo de esta información reciente para el estudio de la respuesta sísmica de edificaciones en general y de mampostería en particular.

El comportamiento observado de estructuras durante las dos últimas décadas ha llevado a que la comunidad internacional relacionada con la ingeniería sismorresistente, haya revisado este comportamiento y como consecuencia sugerido el empleo de un enfoque de diseño sismorresistente diferente al convencional. Este enfoque es conocido como "Diseño sísmico por desempeño", en el cual el desplazamiento lateral de las edificaciones (o deformaciones relativas de entrepiso) es un parámetro relevante en los procedimientos que se emplean para llevar a cabo el proceso de evaluación o diseño por sismo de la estructura. En este trabajo el autor evalúa la capacidad de deformación de especimenes de mampostería sometidos a cargas laterales en ensayes en laboratorio, y demuestra que la dispersión de los resultados encontrados es menor que la de resultados en los que se evalúa la capacidad lateral resistente de estos especimenes.

Por otro lado, en el enfoque de evaluación y diseño por sismo que se propone para edificaciones de mampostería, ú otras con diferente tipo de material, es de suma importancia el empleo de algún parámetro que se pueda relacionar con diferentes niveles de desempeño de la estructura durante sismos. Es decir, es deseable contar con una medida de daño por sismo en la estructura, de manera que el ingeniero, o el propietario, decida si lo considera aceptable ó no. Con esta medida de daño, además de relacionarla a un nivel de desempeño en la estructura, sería posible definir diversas características de la estructura que lleven a minimizar el daño por sismo en ella. Esta última característica es útil no sólo en el proceso de diseño por sismo de estructuras nuevas, sino también para la evaluación por sismo de estructuras existentes, con el objeto de poder tomar decisiones para su rehabilitación sísmica si así fuera necesario.

Una medida de daño por sismo en estructuras con las características anteriores ha sido propuesta por el autor (Rodríguez, 1994; Rodríguez y Aristizábal, 1999), con aplicación principalmente a edificaciones de concreto reforzado. En este trabajo se emplea esta medida para los casos de edificaciones de mampostería, de tabique recocido y de adobe. Tanto para la evaluación de esta medida, como para la evaluación de demandas de deformaciones laterales en edificaciones de mampostería, en este trabajo se emplean algunos registros de aceleraciones que se consideran representativos de movimientos del terreno en algunos sismos ocurridos en México (1985, 1997, 1999), entre ellos algunos en la costa del Pacífico que han causado daños en esa zona en edificaciones de mampostería. Además, con el propósito de evaluar la medida de daño propuesta, así como el potencial de daños de algunos sismos, se emplean registros de aceleraciones obtenidos en sismos ocurridos en diversas partes del mundo, en los que también se 
han observado daños en edificaciones de mampostería. Este último grupo de registros se ha obtenido en terremotos ocurridos en Chile (1985), Japón (1995) y Perú (2001).

\section{CARACTERÍSTICAS DE LOS TERREMOTOS Y REGISTROS DE ACELERACIONES ESTUDIADOS. COMPORTAMIENTO OBSERVADO EN EDIFICACIONES DE MAMPOSTERÍA EN ESTOS EVENTOS}

En este trabajo se emplean registros de aceleraciones e información de daños globales de edificaciones de mampostería obtenidos en siete terremotos ocurridos en distintas partes del mundo. Estos terremotos son:

a) En México, el sismo de Michoacán, el 19-09-1985; de Caleta de Campos, el 11-011997; y el de Oaxaca, el 30-09-1999.

b) En Chile, el sismo del 3-03-1985.

c) En Japón, el sismo de Hyogoken-Nanbu, en Kobe, el 17-01-1995.

d) El sismo de Moquegua, Perú, el 23-06-2001.

Tabla 1. Características de los terremotos y registros de aceleración estudiados

\begin{tabular}{|c|c|c|c|c|c|c|c|c|c|}
\hline Terremoto & Registro & COMP. & Clave & Tipo de suelo & $\begin{array}{l}\text { DISTEPIC } \\
(\mathrm{Km})\end{array}$ & $\begin{array}{c}\mathrm{Ms} \\
(\mathrm{Mw})\end{array}$ & IMM & $\begin{array}{c}\mathrm{A}_{\max } \\
(\mathrm{g})\end{array}$ & $\begin{array}{c}\mathrm{I}_{\mathrm{A}} \\
(\mathrm{m} / \mathrm{s})\end{array}$ \\
\hline MEXICO & SCT & E00W & SCT & Blando & 400 & 8.1 & VIII-IX & 0.17 & 2.33 \\
\hline \multirow[t]{3}{*}{$\begin{array}{c}\text { (Michoacán) } \\
\text { 19-IX-1985 }\end{array}$} & $\begin{array}{l}\text { Caleta de } \\
\text { Campos }\end{array}$ & S00E & CALE-85 & Firme (andesítico) & 400 & 8.1 & VIII-IX & 0.14 & 0.66 \\
\hline & La Union & S00E & & $\begin{array}{c}\text { Firme } \\
\text { ( Volcánica) }\end{array}$ & 400 & 8.1 & VIII-IX & 0.17 & 1.02 \\
\hline & Villita & S00E & & $\begin{array}{c}\text { Firme } \\
\text { (Tonalita) }\end{array}$ & 400 & 8.1 & VIII-IX & 0.13 & 0.42 \\
\hline \multirow[t]{3}{*}{$\begin{array}{c}\text { MEXICO } \\
\text { (Caleta de Campos) } \\
\text { 11-I-1997 }\end{array}$} & $\begin{array}{l}\text { Caleta de } \\
\text { Campos }\end{array}$ & S90E & CALE-97 & $\begin{array}{c}\text { Firme } \\
\text { (andesítico) }\end{array}$ & 10 & $(7.1)$ & - & 0.40 & 2.06 \\
\hline & La Unión & S90E & & Firme & 110 & $(7.1)$ & - & 0.08 & 0.07 \\
\hline & Villita & S90E & & Firme & 50 & $(7.1)$ & - & 0.10 & 0.14 \\
\hline $\begin{array}{r}\text { OAXACA } \\
\text { 30-IX-1999 } \\
\end{array}$ & Río Grande & N90W & RIOG-99 & Firme (Roca) & 50 & $(7.5)$ & VIII & 0.30 & 1.84 \\
\hline $\begin{array}{l}\text { CHILE } \\
\text { 3-III-1985 }\end{array}$ & LLOLLEO & $\mathrm{N} 10 \mathrm{E}$ & LLO & Arenoso & 45 & 7.8 & VIII & 0.67 & 15.1 \\
\hline $\begin{array}{c}\text { HYOGOKEN-NANBU } \\
\text { JAPON } \\
\text { 17-I-1995 } \\
\end{array}$ & $\begin{array}{c}\text { KOBE } \\
\text { JMA }\end{array}$ & NOOE & КOB & Deposito aluvial & 10 & 6.9 & VIII-IX & 0.84 & 8.35 \\
\hline $\begin{array}{c}\text { AREQUIPA, PERU } \\
\text { 23-VI-2001 }\end{array}$ & Moquegua & E00W & MOQ & Conglomerado & - & 7.9 & VII-VIII & 0.30 & 3.37 \\
\hline
\end{tabular}

La tabla 1 (Rodríguez y Aristizábal, 1999; Rodríguez et al., 1998; Singh et al., 2000; Lermo et al., 2002) muestra un resumen de algunas características de los terremotos estudiados, 
así como de los registros sísmicos que se analizan en este estudio. En esta tabla se indican características de estos terremotos tales como magnitud (medida en las escalas $\mathrm{M}_{\mathrm{s}} \mathrm{o} \mathrm{M}_{\mathrm{w}}$ ) é intensidades de Mercalli (IMM) máximas observadas. En los registros que se incluyen en la tabla 1, se identifica la componente seleccionada para el estudio, el tipo de suelo en que se ubica el acelerómetro, la distancia de éste al epicentro, así como la aceleración máxima registrada en el terreno asociada al registro y la intensidad de Arias calculada para el registro respectivo. La fig 1 muestra, empleando la misma escala, algunos de los registros de aceleraciones que se incluyen en la tabla 1. En lo que sigue se dan mas detalles de los terremotos que se incluyen en la tabla 1 y de sus efectos en edificaciones de mampostería, así como características relevantes de los espectros de respuesta de algunos de los registros empleados.

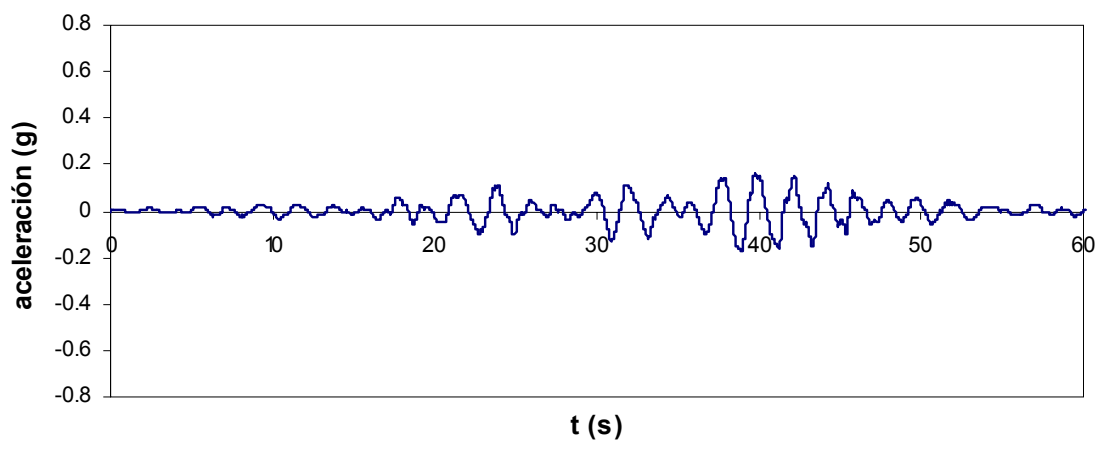

a) SCT 1985, E00W

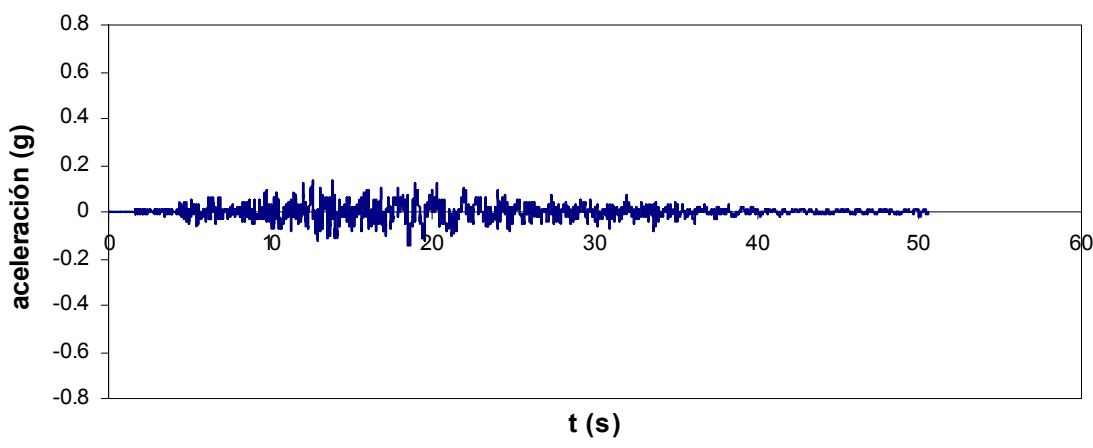

b) Caleta de Campos 1985, S00E

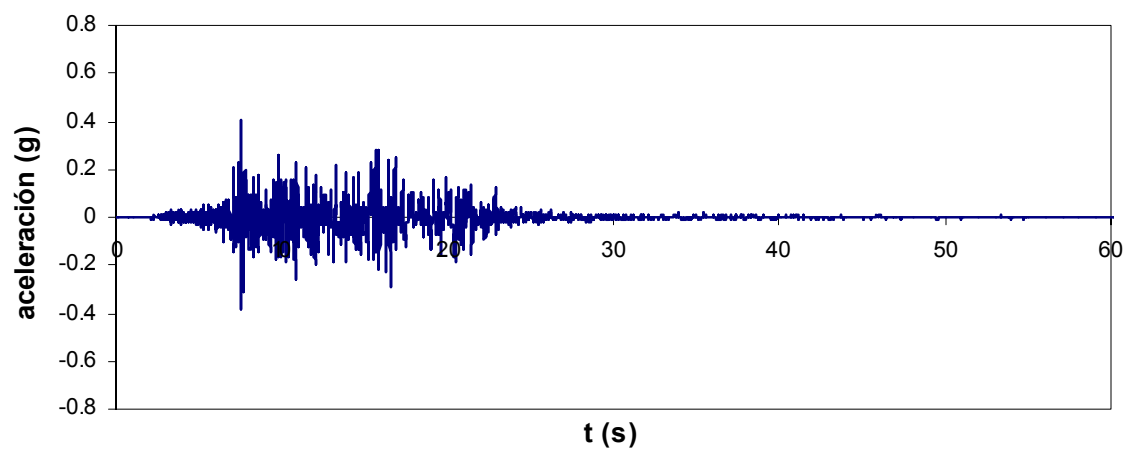

c) Caleta de Campos 1997, S90E

Figura 1. Registros de aceleraciones 


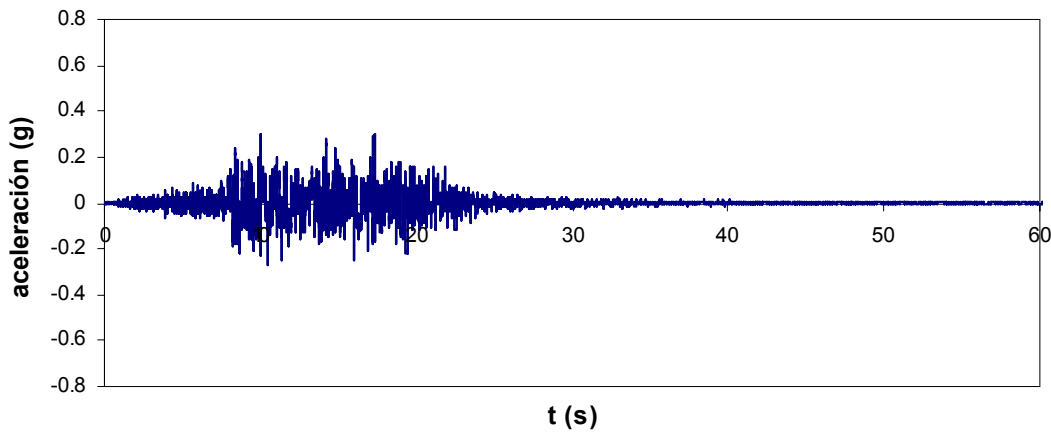

d) Río Grande 1999, N90W

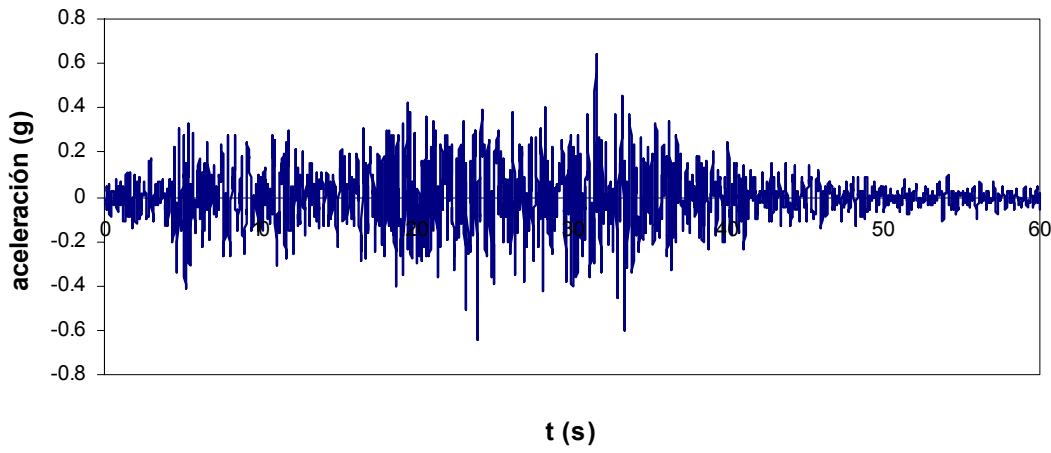

e) LLolleo 1985, N10E

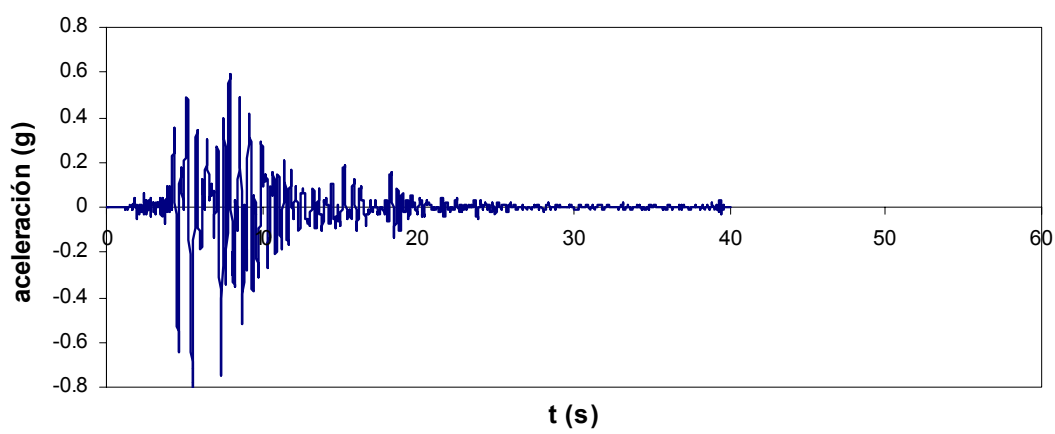

f) Kobe 1995, N00E

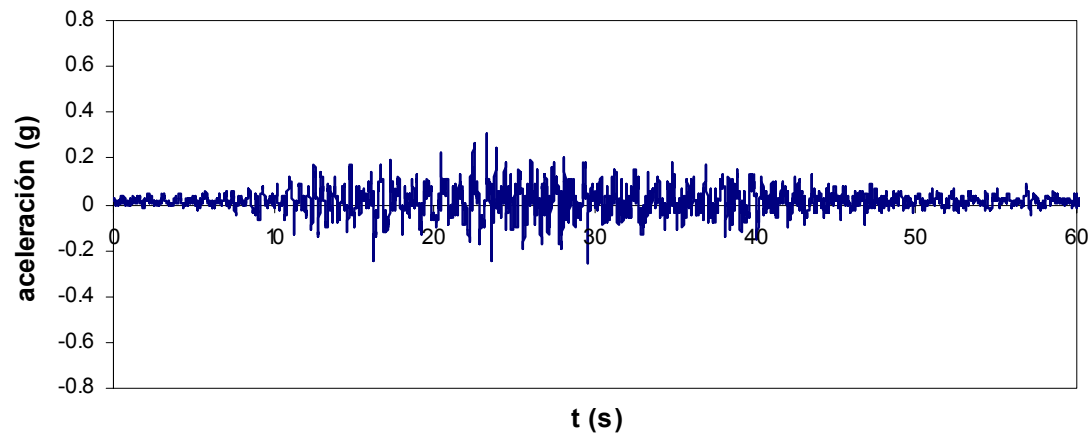

g) Arequipa 2001, E00W

Figura 1. Registros de aceleraciones (continuación) 


\section{Terremotos en México en 1985, 1997 y 1999}

La información de daños en la zona epicentral del terremoto de Michoacán del 19 de septiembre de 1985 es escasa y no existen estadísticas de daños o colapsos en esta zona, lo que contrasta con la extensa información sobre el tema en la ciudad de México (Rosenblueth y Ruiz, 1989; NZ Reconnaissance Team, 1988). A pesar de lo anterior se acepta que la mayor parte los daños o colapsos ocurrieron en el DF, con mínimo daño en edificaciones a base de mampostería en esta ciudad. La fig 2 muestra la zona de ruptura del terremoto de 1985, así como la ubicación de algunas poblaciones cercanas a esta zona de ruptura. Este terremoto fue típico de uno de subducción del tipo interplaca, en este caso de subducción de la Placa de Cocos dentro del continente.

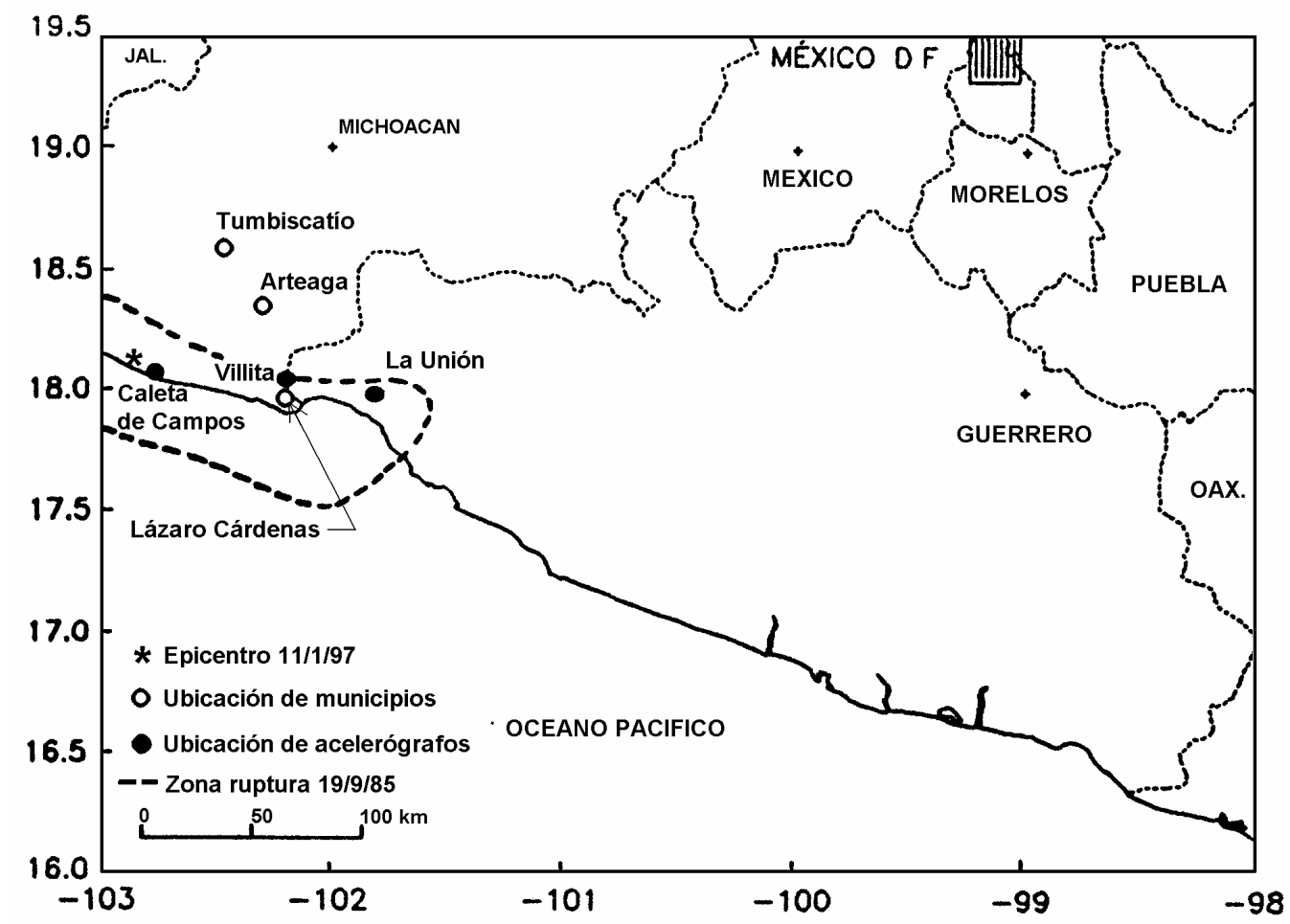

Figura 2. Ubicación de estaciones y epicentros de los sismos de Michoacán de 1985 y 1997

El 11 de enero de 1997 ocurrió el sismo de Caleta de Campos, la ubicación del epicentro de este sismo se muestra en la fig 2. Este sismo se ubicó dentro de la zona de ruptura de la Placa de Cocos que originó el sismo de 1985. Es de interés que el sismo de Caleta de Campos de 1985 fue de naturaleza diferente al de 1985, ya que se clasifica como de tipo intraplaca (Anderson et al., 1997; Singh et al., 2000). El tipo de mecanismo que origina el tipo de sismos de subducción del tipo intraplaca se interpreta como provocado por deformaciones internas de la placa que se desliza bajo el continente (Anderson et al., 1997), y no ocurre necesariamente cerca al borde de la interfase de las placas, como es el caso de un sismo de subducción (Singh et al., 2000). 
Rodríguez et al. (1998) han analizado algunos registros de aceleraciones obtenidos tanto en el terremoto de Caleta de Campos de 1997, así como en el sismo de Michoacán de 1985. Es de interés el análisis de registros de aceleraciones obtenidos en las mismas estaciones en la zona epicentral de los dos últimos terremotos mencionados. Estas estaciones son Caleta de Campos, La Unión y Villita, cuyas ubicaciones se ilustran en la fig 2. Como se aprecia en esta figura la estación Caleta de Campos se ubica cerca al epicentro del terremoto de 1997. La tabla 2 (Rodriguez et al., 1998) muestra las aceleraciones máximas registradas en la estación Caleta de Campos en 1997, así como las intensidades de Arias (Arias, 1970) correspondientes. Esta medida de intensidad se considera también como una medida del potencial destructivo asociado a un registro de aceleraciones. En la tabla 2 se observa que en esta estación estos parámetros son mayores para los registros de 1997 respecto a los de 1985. Por el contrario, estos parámetros en las estaciones de La Unión y Villita son menores para los registros de 1997 si se compara con los de 1985. Los resultados de intensidad de Arias para estos registros, que se muestran en la tabla 2, permiten distinguir sus diferentes niveles de potencial destructivo, en particular confirmar que el potencial destructivo del registro de Caleta de Campos de 1997 es bastante mayor que el correspondiente al registro en el mismo lugar en 1985.

Tabla 2. Características de los registros analizados

\begin{tabular}{|c|c|c|c|c|c|c|c|c|}
\hline \multirow{2}{*}{ Estación } & \multicolumn{4}{|c|}{ Amáx/g } & \multicolumn{4}{c|}{ IA (m/s) } \\
\cline { 2 - 9 } & \multicolumn{2}{|c|}{1997} & \multicolumn{2}{|c|}{1985} & \multicolumn{2}{c|}{1997} & \multicolumn{2}{c|}{1985} \\
\cline { 2 - 9 } & S 90 E & S 00 E & S 90 E & S 00 E & S 90 E & S 00 E & S 90 E & S 00 E \\
\hline Caleta de Campos & 0.40 & 0.36 & 0.14 & 0.14 & 2.06 & 1.60 & 0.47 & 0.66 \\
La Unión & 0.08 & 0.08 & 0.15 & 0.17 & 0.07 & 0.05 & 0.90 & 1.02 \\
Villita & 0.10 & 0.11 & 0.13 & 0.13 & 0.14 & 0.09 & 0.28 & 0.42 \\
\hline
\end{tabular}

Rodríguez et al. (1998) han analizado estadísticas de daños de vivienda de mampostería observados en el terremoto de Caleta de Campos de 1997, y han destacado la alta incidencia de daños en este tipo de edificaciones, particularmente en las de adobe. En general estos daños fueron mayores que los observados en el sismo de Michoacán de 1985. Si se consideran estas observaciones, y que la magnitud del terremoto de Michoacán fue bastante mayor que la del terremoto de Caleta de Campos de 1997, ver tabla 1, así como los resultados de la tabla 2, se pone en evidencia la importancia del tipo de mecanismo de falla que origina un terremoto. De acuerdo con estos resultados, en la zona epicentral un terremoto de subducción del tipo intraplaca llevaría a niveles de riesgo sísmico en esta zona mayores que los correspondientes a uno de magnitud comparable de subducción del tipo interplaca.

Otro evento de interés para la ingeniería sismorresistente de edificaciones de mampostería es el terremoto de Oaxaca del 30 de septiembre de 1999. La fig 3 muestra el epicentro de este terremoto, así como la distribución de isosistas de intensidades de este evento (Singh et al., 2000). Este terremoto se clasifica como de subducción del tipo intraplaca. De acuerdo con estadísticas de daños en este terremoto en el estado de Oaxaca, más de 41,000 viviendas fueron afectadas por el terremoto, y por ejemplo, sólo en San Pedro Tututepec en la costa del Pacífico, 
cerca de la zona epicentral ( fig 3), 3,000 viviendas llegaron al colapso y 3,200 tuvieron algún tipo de daño (Singh et al. , 2000).

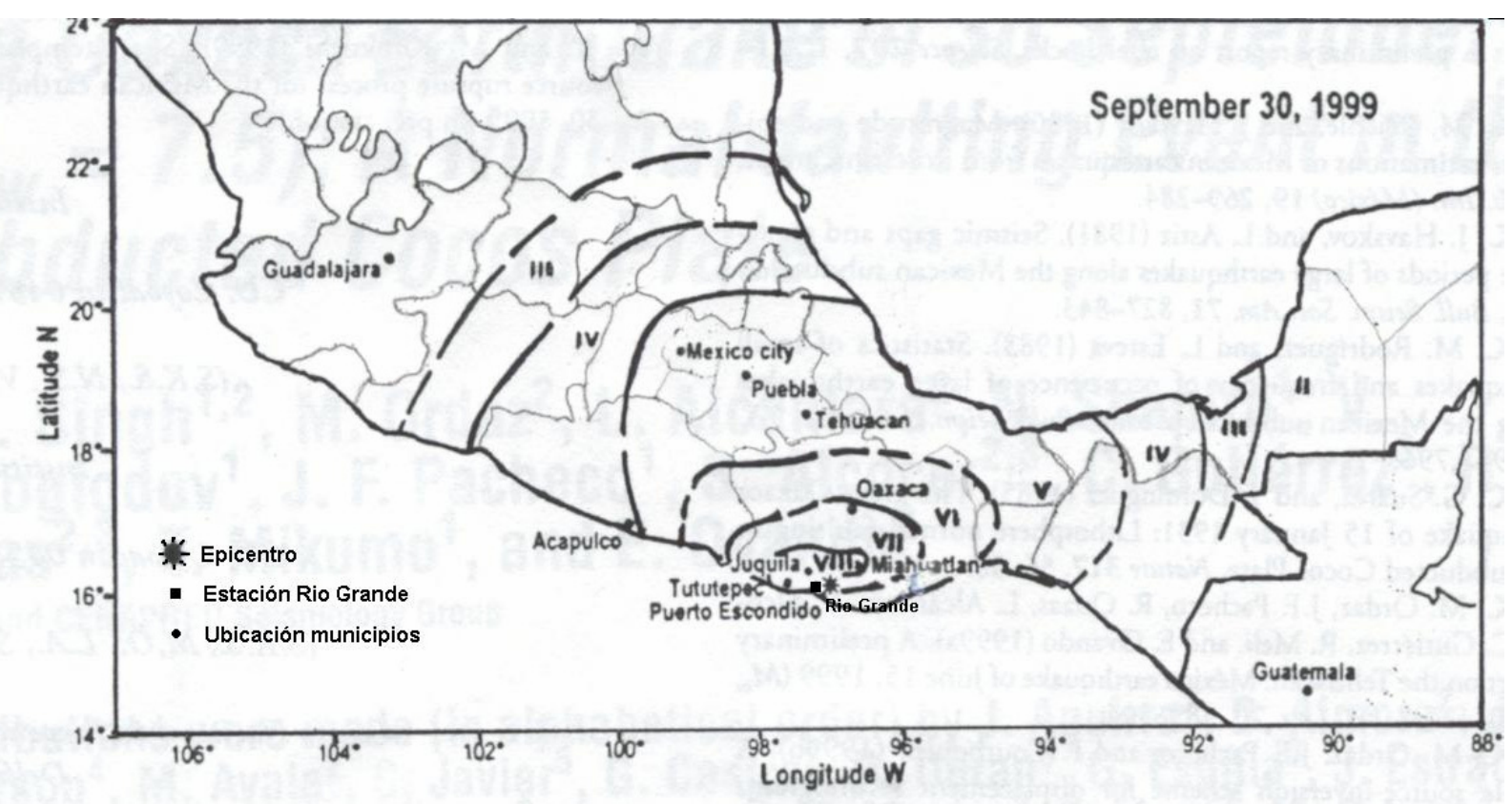

Figura 3. Epicentro e isosistas del sismo de Oaxaca del 30 de septiembre de 1999

Para propósitos de estudio de la respuesta sísmica de edificaciones de mampostería en el terremoto de Oaxaca de 1999 se seleccionó el registro de aceleraciones componente N90W obtenido en la estación Río Grande (RIOG-99), ubicado cerca de la zona epicentral ( fig 3).

Como se deduce de los resultados de este estudio, así como de observaciones de sismos del tipo de intraplaca anteriores en México (Singh et al., 2000), e incluso en otras zonas sísmicas, por ejemplo en Chile (Astroza et al., 2002), los daños en edificaciones provocados por este tipo de sismo en las zonas cercanas al epicentro son mayores que los correspondientes a sismos de magnitud semejantes pero del tipo de subducción de interplaca. En particular en México es de relevante la experiencia del terremoto de Oaxaca de 15 de enero de 1931, el cual causó una gran destrucción en el estado de Oaxaca, particularmente en la ciudad de Oaxaca, y el cual también se clasifica como de subducción del tipo intraplaca (Singh et al., 2000). De acuerdo con Singh et al. (2000), eventos como el de Oaxaca de 1931 demuestran que los terremotos del tipo intraplaca en México son de mayor energía en altas frecuencias que terremotos comparables pero de subducción del tipo interplaca. Esto explicaría los extensos daños en edificaciones de mampostería observados en sismos intensos anteriores en zonas del altiplano en México causados por este tipo de sismos. Esta observación se complementa con resultados encontrados por Astroza et al. (2002), quienes con base en daños observados en viviendas de adobe en el terremoto de Punitaqui del 14 de octubre de 1997 en el norte de Chile, sugieren que las fórmulas de atenuación deben de diferenciar si los sismos son del tipo interplaca o intraplaca. 


\section{Terremoto de Chile, marzo 3, 1985}

Este terremoto se originó en el mar a unos $20 \mathrm{~km}$ de la costa chilena, entre las localidades de Valparaíso y Algarrobo, por la subducción de la placa de Nazca en el continente. Se considera que este terremoto ha sido uno de los más importantes del siglo XX, comparable al terremoto de Valparaíso en 1906 (Saragoni et al., 1993). Es de interés que en este terremoto la mayoría de los daños de gravedad en viviendas ocurrieron en casas de adobe en zonas rurales o en mampostería sin refuerzo de barrios antiguos de ciudades. En particular es relevante el número de edificaciones de adobe que llegaron al colapso en la zonas de LLolleo (Flores, 1993). Por este motivo se decidió emplear en este estudio el registro de LLolleo, componente N10E, con aceleración máxima del terreno igual a $0.67 \mathrm{~g}$.

\section{Terremoto de Japón, Kobe, enero 17, 1995}

El terremoto Hyogo-Ken-Nambu del 17 de enero de 1995 es considerado el más destructivo en Japón desde el que afectó a las ciudades de Tokyo y Yokohama en 1923. Este evento se originó bajo el océano, en la bahía de Osaka, entre la ciudad de Kobe y la isla de Awaji. De este terremoto, para este estudio se eligió el registro obtenido en la ciudad de Kobe, en el Observatorio JMA, y se seleccionó la componente N00E, con una aceleración máxima del terreno de $0.82 \mathrm{~g}$. Este terremoto afectó muchos tipos de estructuras principalmente en la ciudad de Kobe (Muguruma et al., 1995). En lo referente a efectos de este terremoto en edificaciones para vivienda en la ciudad de Kobe, destaca la gran destrucción de viviendas tradicionales japonesas, construidas con marcos de madera, de paredes débiles y techos pesados, desfavorable desde el punto de vista sísmico por las altas fuerzas sísmicas que se generan durante un sismo asociadas a las masas importantes de los techos. A pesar de que esta práctica constructiva de vivienda es bastante diferente a la de mampostería a que se refiere este estudio, las altas aceleraciones del registro JMA mencionado, así como el alto índice daños observados en diferentes tipos de estructuras en este evento hacen que sea de interés el estudio del potencial destructivo de este registro en edificaciones de mampostería.

\section{Terremoto de Arequipa, Perú, junio 23, 2001}

Este terremoto se originó en la costa del Pacífico, en el sur del Perú, por subducción de la Placa de Nazca dentro del continente. En este evento destaca el alto valor de su magnitud, Ms=7.9. La ubicación del epicentro de este evento así como la distribución de isosistas de intensidades se muestra en la fig 4 (Muñoz y Timan, 2001). La única estación que registró las aceleraciones de este evento se ubica en la ciudad de Moquegua, fig 4, a aproximadamente $320 \mathrm{Km}$ del epicentro y localizada sobre depósitos sedimentarios (conglomerado), Lermo et al. (2002).

Respecto a los daños causados por este terremoto en viviendas, en este estudio se hace referencia sólo a los observados en la ciudad de Moquegua, por la correlación que podría haber con los análisis que aquí se llevan a cabo con el registro mencionado. De la evaluación de daños en edificaciones de mampostería en Moquegua, destaca la gran destrucción de viviendas de adobe, así como de tabique de arcilla con deficiencias constructivas o estructurales (Zegarra et 
al., 2001). Parte de estas viviendas se ubicaban en zonas periféricas de la ciudad y se localizaban en zonas de topografía con pendientes fuertes, sugiriendo efectos de sitio (Lermo et al., 2002).

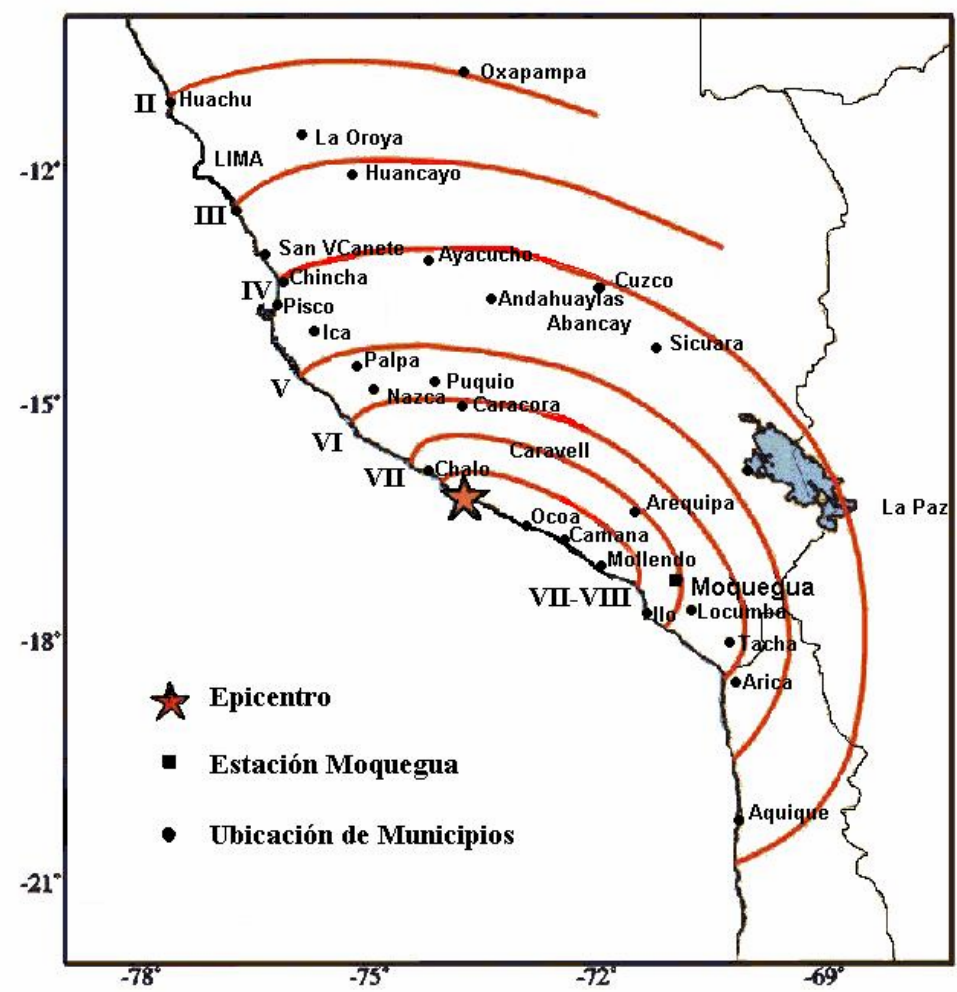

Figura 4. Epicentro e isosistas del sismo del Perú del 23 de junio del 2001

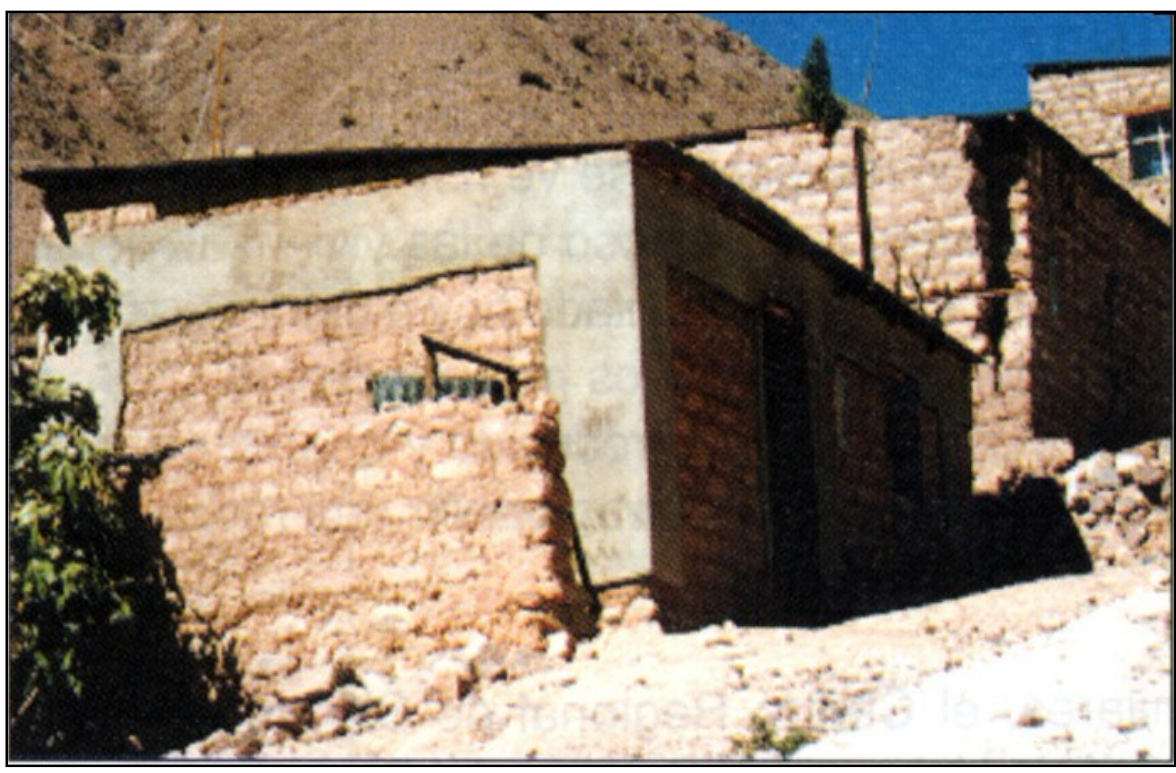

Figura 5. Vivienda de adobe rehabilitada con aplanado de mortero sobre malla electrosoldada que resistió sin daños el terremoto de Arequipa (Zegarra et al., 2001) 
Aún cuando se trata de un número muy limitado de casos, es de interés mencionar el comportamiento observado en este terremoto en viviendas de adobe que fueron reforzadas antes del evento sísmico. Las viviendas a que se hace referencia fueron reforzadas con aplanados de mortero sobre mallas de alambre electrosoldadas, formando franjas verticales y horizontales en la periferia de los muros como se aprecia en la fig 5 (Zegarra et al., 2001). De acuerdo con una evaluación de daños en Moquegua, las viviendas que tuvieron el reforzamiento mencionado mostraron un buen comportamiento, mientras que viviendas vecinas de adobe con construcción convencional mostraron daños o colapsos (Zegarra et al., 2001).

\section{UN ENFOQUE ALTERNATIVO DE DISEÑO SÍSMICO DE EDIFICACIONES DE MAMPOSTERÍA}

El procedimiento tradicional de diseño sismorresistente se basa en criterios en los que la resistencia de la estructura es un parámetro básico de diseño. A raíz de daños importantes o colapsos observados en estructuras en años recientes, aún en sismos que se podrían considerar moderados, en diversas partes del mundo ha surgido el interés de emplear criterios alternativos de diseño sísmico que lleven a reducir de manera importante la vulnerabilidad de estructuras resistentes a sismos. Un ejemplo de estos criterios son las recomendaciones de diseño para la rehabilitación de edificaciones en zonas sísmicas en los Estados Unidos (FEMA 273, 1997). Este criterio alternativo, conocido también con el nombre de "Diseño sísmico por desempeño", aún no se encuentra incorporado en reglamentos de diseño sísmico; sin embargo, es muy probable que lo sea en el futuro.

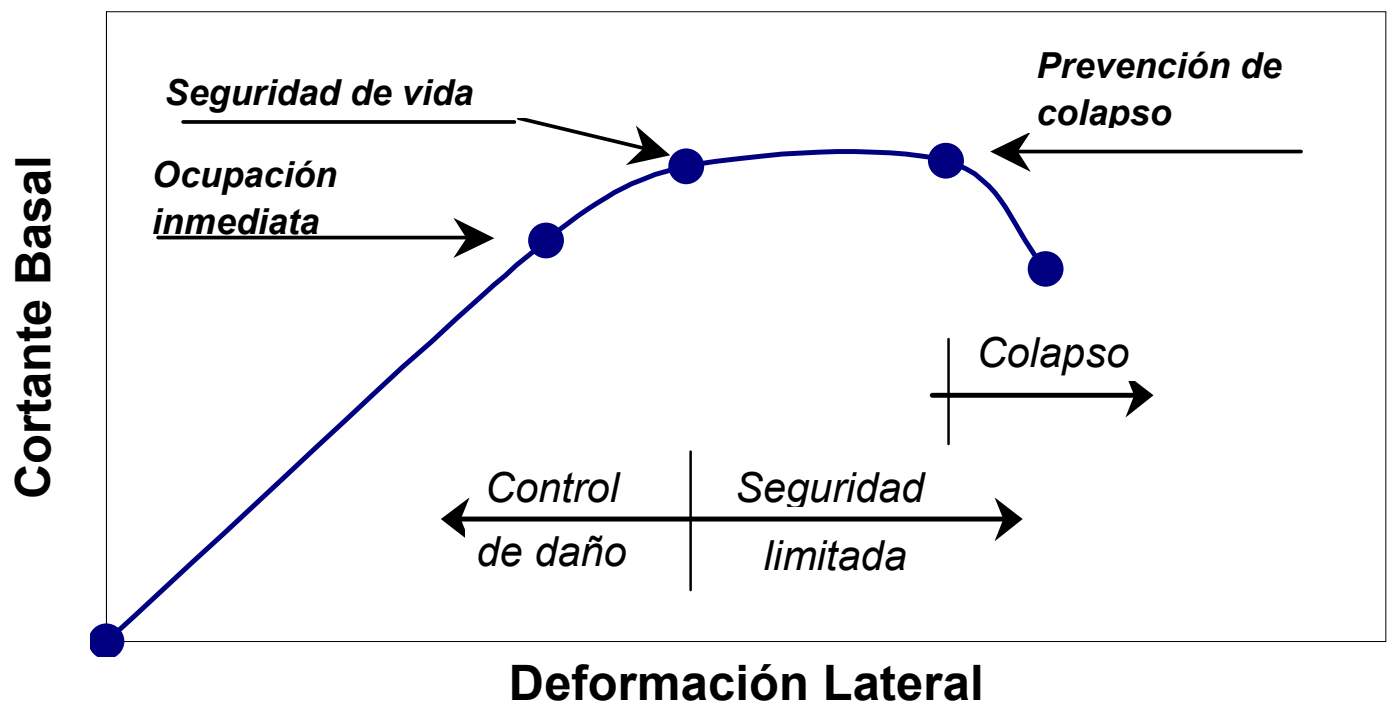

Figura 6. Demandas de niveles de comportamiento y deformación en estructuras poco dúctiles

El documento FEMA 273 recomienda considerar tres niveles de comportamiento sísmico: a) Ocupación inmediata, b) Seguridad de vida, y c) Prevención de colapso. Estos niveles de comportamiento se ilustran en la fig 6, en este caso para estructuras con ductilidad limitada. Se debe mencionar que los niveles de comportamiento mencionados están relacionados a diferentes niveles de peligro sísmico. Por ejemplo, de acuerdo con FEMA 273, edificaciones importantes 
(tales como escuelas, estaciones de bomberos, etc.) se debieran diseñar para el nivel de ocupación inmediata asociado a un periodo de recurrencia de 475 años. Para estas mismas edificaciones, de acuerdo con FEMA 273, el nivel de seguridad de vida se debe asociar a un periodo de recurrencia de 2475 años

\section{Criterios de diseño sísmico por desempeño en edificaciones de mampostería}

De acuerdo con el documento FEMA 273, para las edificaciones de mampostería se deben considerar los tres niveles de comportamiento sísmico aquí descritos. Sin embargo, el autor sugiere considerar en este enfoque alternativo sólo los niveles de comportamiento de seguridad de vida y de prevención de colapso sugeridos por FEMA 273. La variabilidad de la respuesta sísmica de edificaciones de mampostería, principalmente por las características de sus materiales, no sugiere el empleo de procedimientos de diseño elaborados para este tipo de edificación, como el propuesto por FEMA 273.

La definición del nivel de seguridad de vida depende en realidad de la cantidad de daño estructural y no estructural en la edificación que podría ser aceptado por el propietario de ésta. En opinión del autor este nivel pudiera estar asociado a daños reparables en la estructura. En una evaluación de la capacidad de deformación lateral de especimenes de mampostería que se lleva a cabo en este trabajo, el nivel de comportamiento de prevención de colapso, como se describe más adelante se define como el nivel de respuesta de la estructura en el que la capacidad lateral se reduce un $20 \%$.

\section{Evaluación del nivel de comportamiento de prevención de colapso en muros de mampostería confinada construida con unidades sólidas de arcilla}

Rodríguez et al. (2000) han evaluado resultados del comportamiento experimental ante cargas laterales cíclicas de nueve especimenes de muros de mampostería confinada, construidos con ladrillos sólidos de arcilla, seis de estos especimenes fueron ensayados en México y los restantes en Colombia y Chile. La tabla 3 (Rodríguez et al., 2000) muestra las características más relevantes de los especimenes estudiados.

Para el estudio del comportamiento asociado al nivel de prevención de colapso en los especimenes estudiados se evaluó la resistencia lateral experimental máxima, $V_{\max }$, a partir de las envolventes de las curvas carga-deformación lateral obtenidas en los ensayes de estos especimenes. El nivel de capacidad de deformación de entrepiso máximo en los especimenes, $d_{2}$, se definió como la distorsión de entrepiso asociada a una disminución del $20 \%$ de la capacidad lateral del espécimen, lo que se ilustra en la fig 7.

Los resultados de aplicar los conceptos anteriores para los especimenes estudiados se muestran gráficamente en la fig 8 (Rodríguez et al., 2000). Esta figura muestra las deformaciones experimentales máximas, con la definición dada, en función de la relación de esbeltez $h / l$ de los especimenes, donde $h$ es la altura del muro y $l$ su longitud. De acuerdo con 
estos resultados, la media de $d_{2}$ es igual a 0.0052 , con un coeficiente de variación (CV) igual a 0.08 .

Tabla 3: Características de especimenes de mampostería confinada

\begin{tabular}{|c|c|c|c|c|c|c|c|}
\hline \multirow[b]{2}{*}{ Espécimen } & \multirow[b]{2}{*}{ Ref } & \multicolumn{2}{|c|}{ Dimensiones } & \multicolumn{2}{|c|}{ Castillos } & \multicolumn{2}{|c|}{ Dalas } \\
\hline & & $\begin{array}{l}\mathrm{L} \times \mathrm{h} \\
(\mathrm{m})\end{array}$ & $\begin{array}{c}\text { Espesor } \\
\text { (m) }\end{array}$ & $\begin{array}{c}\text { Dimensiones } \\
\text { a1 x a2 } \\
(\mathrm{m})\end{array}$ & $\begin{array}{c}\text { Refuerzo } \\
\text { Longitud. } \\
(\mathrm{mm})\end{array}$ & $\begin{array}{c}\text { Dimensiones } \\
\text { a1 x a2 } \\
(\mathrm{m})\end{array}$ & $\begin{array}{c}\text { Refuerzo } \\
\text { Longitud. } \\
(\mathrm{mm})\end{array}$ \\
\hline $\mathrm{S} / \mathrm{I}$ & Meli (1975) & $1.80 \times 1.80$ & 0.14 & ------- & -------- & -------- & ------- \\
\hline 902 & $\begin{array}{l}\text { Meli y Salgado } \\
\text { (1969) }\end{array}$ & $2.40 \times 2.40$ & 0.12 & $0.12 \times 0.15$ & $4 \phi 12.7$ & $0.12 \times 0.20$ & ------- \\
\hline $3 \mathrm{D}$ & Ruiz (1995) & $2.00 \mathrm{z} 2.50^{*}$ & 0.125 & $0.125 \times 0.15$ & $4 \phi 9.5$ & $0.125 \times 0.25$ & $4 \phi 12.7$ \\
\hline WBW & $\begin{array}{c}\text { Alcocer } \text { et al } \\
(1994)\end{array}$ & $2.00 \times 2.50^{*}$ & 0.125 & $0.125 \times 0.15$ & $4 \phi 9.5$ & $0.125 \times 0.25$ & $4 \phi 12.7$ \\
\hline WWW & $\begin{array}{c}\text { Alcocer } \text { et al } \\
\text { (1994) }\end{array}$ & $2.00 \times 2.50 *$ & 0.125 & $0.125 \times 0.15$ & $4 \phi 9.5$ & $0.125 \times 0.25$ & $4 \phi 12.7$ \\
\hline MURO 6 & $\begin{array}{c}\text { García y Yamin } \\
\text { (1994) }\end{array}$ & $3.15 \times 2.15$ & 0.12 & $0.12 \times 0.20$ & $3 \phi 12$ & $0.12 \times 0.20$ & $4 \phi 9.5$ \\
\hline $\mathrm{B} 12$ & Herrera (1992) & $2.40 \times 2.40$ & 0.14 & $0.14 \times 0.20$ & $4 \phi 10$ & $0.25 \times 0.20$ & $4 \phi 10$ \\
\hline $\mathrm{B} 2$ & Herrera (1992) & $2.40 \times 2.40$ & 0.14 & $0.14 \times 0.20$ & $4 \phi 10$ & $0.25 \times 0.20$ & $4 \phi 10$ \\
\hline M2 & Aguilar (1994) & $2.40 \times 2.40$ & 0.12 & $0.12 \times 0.15$ & $4 \phi 10$ & $0.12 \times 0.25$ & $4 \phi 12.7$ \\
\hline
\end{tabular}

Notas:

* Longitud promedio

1 Longitud de muro

h Altura de muro

a1 Ancho de elemento de CR

a2 Peralte de elemento de CR

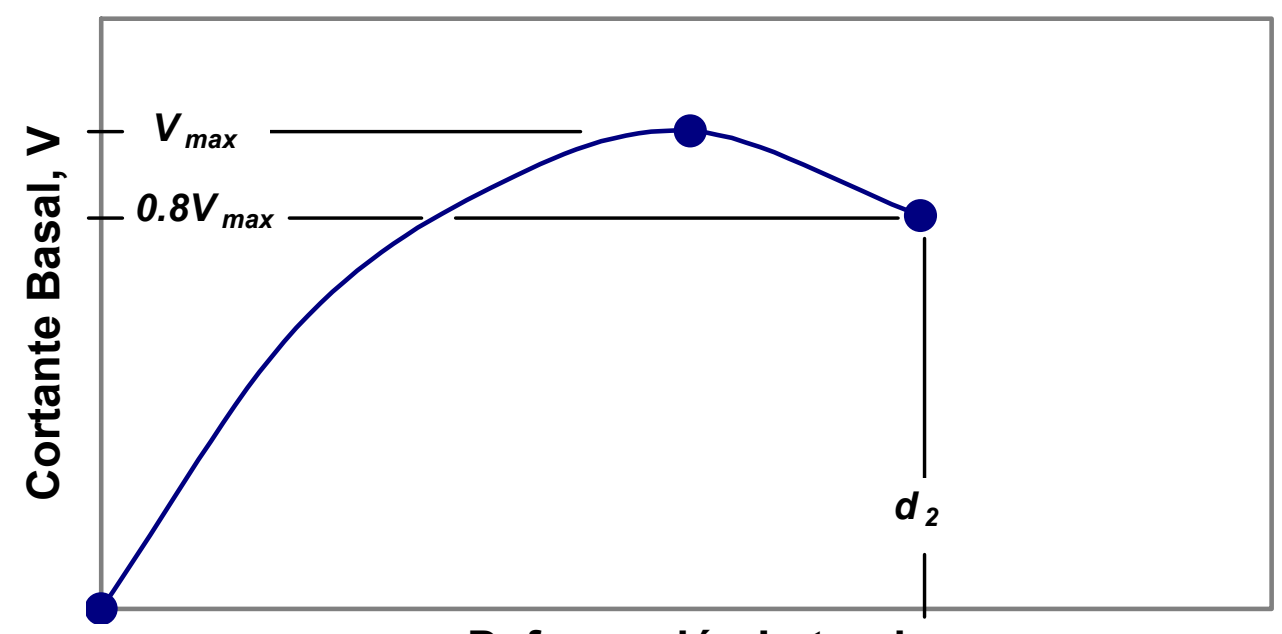

Deformación Lateral

Figura 7. Definición de deformación de entrepiso en el nivel de prevención de colapso 


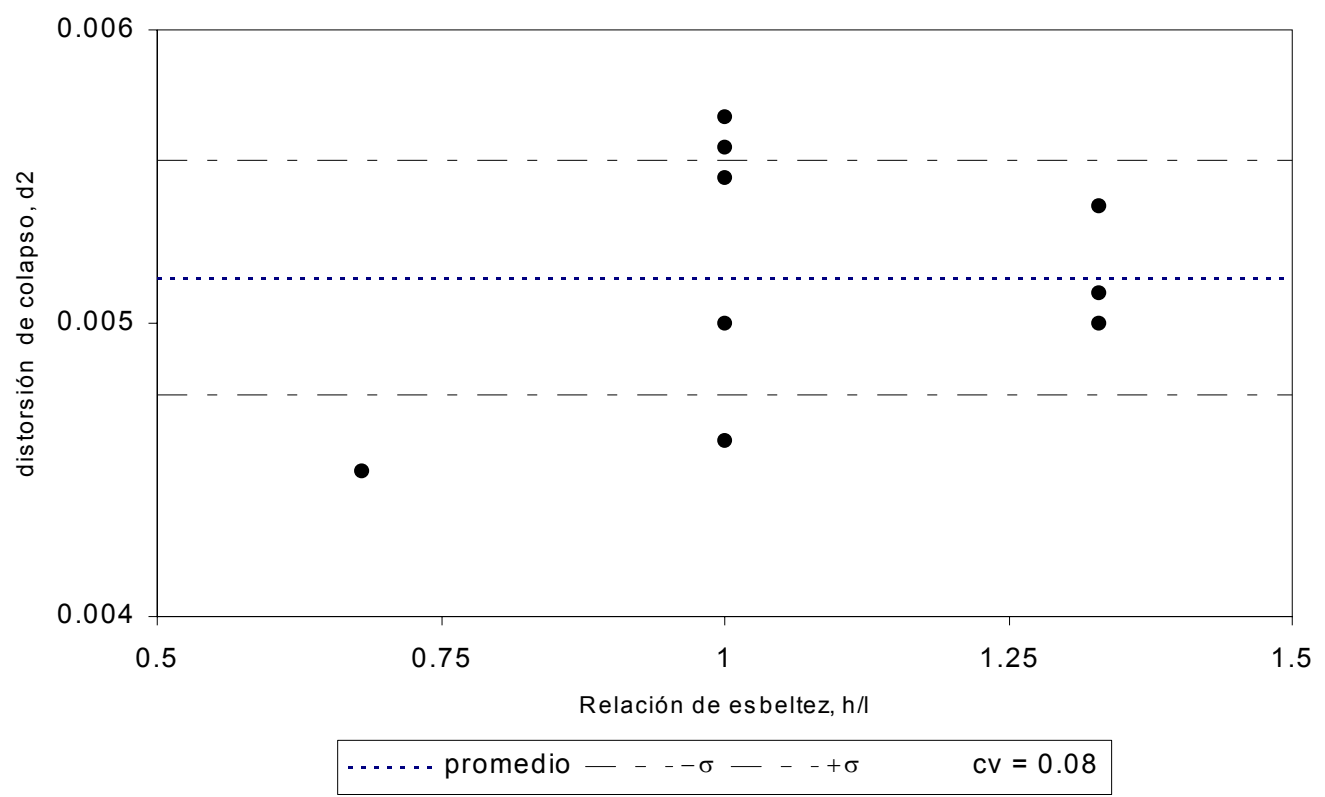

Figura 8. Deformaciones de entrepiso medidas en el nivel de prevención de colapso ( $\sigma=$ desviación estándar)

Con el objeto de evaluar la dispersión de resultados en la predicción de la resistencia lateral de los especimenes estudiados y compararla con la dispersión resultante de la evaluación de capacidad de deformación lateral, Rodríguez et al. (2000) evaluaron la capacidad resistente de los especimenes estudiados empleando las especificaciones del Reglamento de Construcciones del DF (RCDF, 1993), para lo cual emplearon un factor de reducción de resistencia igual a 1.0. Esta resistencia lateral, $V_{R D F}$ se calculó con la expresión:

$$
V_{R D F}=\left(0.5 v^{*} A_{T}+0.3 P\right) \leq 1.5 v^{*} A_{T}
$$

donde $\mathrm{v}^{*}$ es el esfuerzo cortante resistente para mampostería confinada, de acuerdo con el RCDF (1993). Para este parámetro se está considerando un valor de $3 \mathrm{~kg} / \mathrm{cm}^{2}$. El parámetro $A_{T}$ es el área transversal del muro y $P$ es la carga axial aplicada. La fig 9 (Rodríguez et al., 2000) muestra resultados obtenidos empleando este procedimiento para los especimenes estudiados. Como se aprecia en la fig 9, la media de la relación $V_{\max } / V_{R D F}$ es igual a 1.9, con un valor para CV igual a 0.26. Estos resultados muestran que en estos especimenes la predicción de resistencia del RCDF es bastante conservadora y con una dispersión bastante mayor que la asociada a la dispersión experimental de la capacidad de deformación en ellos. Aún cuando el número de especimenes que forman el banco de datos estudiado no es muy grande, estos resultados sugieren la conveniencia del empleo de un criterio de diseño sísmico de edificaciones de mampostería basado en la capacidad de deformación lateral de ellas. 


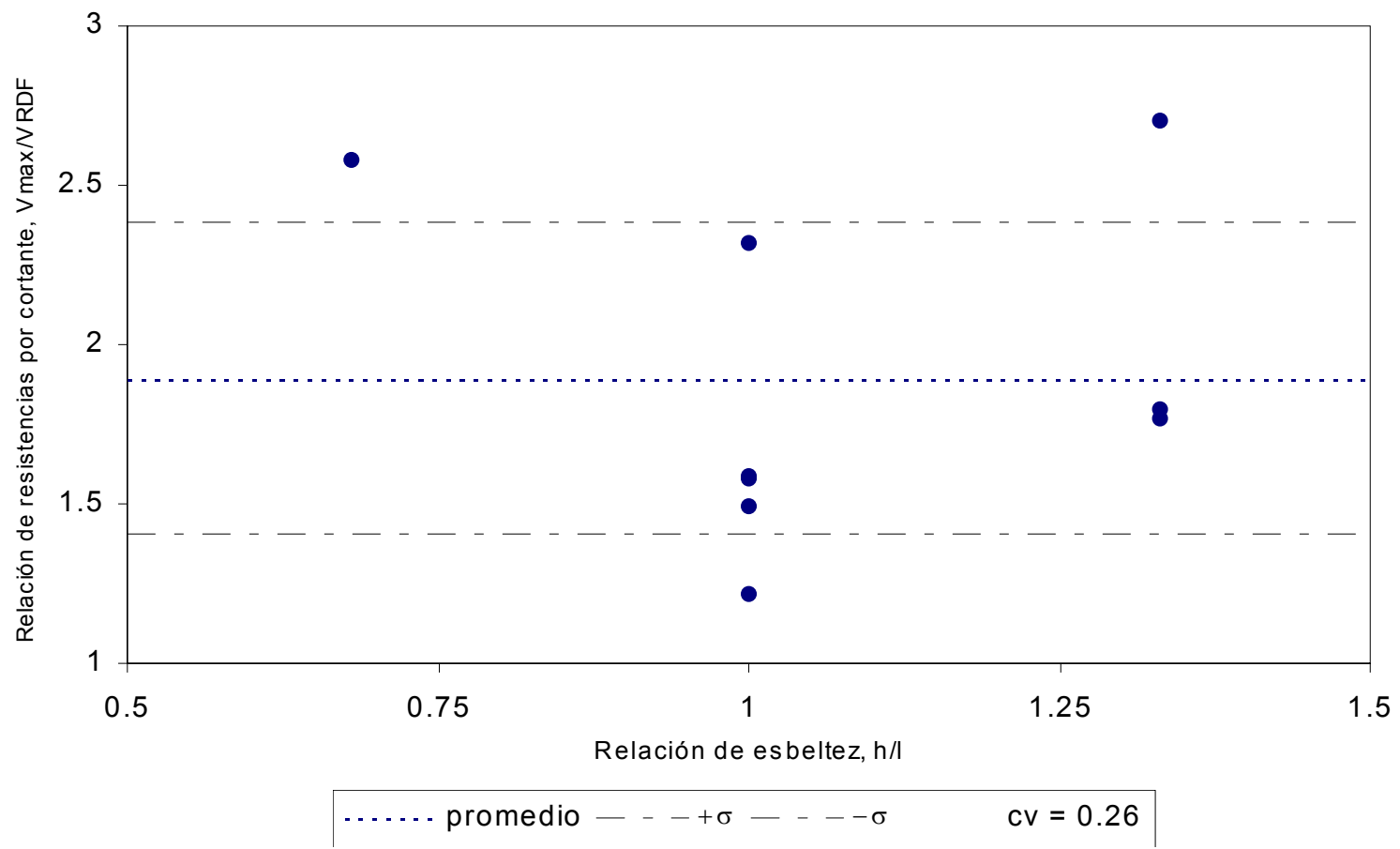

Figura 9. Resistencia por cortante calculada y medida. ( $\sigma=$ desviación estándar)

\section{ANÁLISIS DE LAS DEMANDAS Y CAPACIDADES DE RESISTENCIA Y DESPLAZAMIENTOS LATERALES EN EDIFICACIONES DE MAMPOSTERÍA EMPLEANDO ACELEROGRAMAS DE TERREMOTOS ESTUDIADOS}

En ingeniería sismorresistente es fundamental revisar si en una estructura la capacidad es mayor o al menos igual a la demanda originada por un movimiento sísmico. En lo que sigue, de manera aproximada se efectúa esta revisión en edificaciones de mampostería, empleando algunos de los registros de aceleraciones que se incluyen en la tabla 1. Como parte del enfoque alternativo de diseño sísmico aquí descrito, además de la resistencia en edificaciones de mampostería, en esta revisión se toma en cuenta las demandas y capacidades de deformación lateral de éstas.

\section{Análisis de demandas y capacidades resistentes}

Se evaluaron espectros de pseudoaceleraciones, $\mathrm{Sa} / \mathrm{g}$, correspondientes a siete de los registros descritos en la tabla 1. Estos espectros corresponden a los registros de México seleccionados para esta evaluación. Estos registros son SCT-85, CALE-85, CALE-97 y RIOG-99. Los dos primeros son del terremoto de 1985, y los últimos de los terremotos de Caleta de Campos 1997 y Oaxaca 1999, respectivamente. También se emplea el registro LLO del terremoto de Chile 1995, el registro KOB del terremoto de Kobe 1995, y el registro MOQ del terremoto de Perú 2001.

La fig 10 muestra espectros elásticos de aceleraciones para los registros mencionados, los que se evaluaron considerando una relación de ductilidad de desplazamientos, $\mu$, igual a 1. El 
intervalo de periodos en estos espectros es pequeño, lo que corresponde a periodos fundamentales típicos de edificaciones de mampostería de hasta cinco niveles, como se demuestra más adelante. Aún cuando la relación entre $\mu$ y el llamado factor de comportamiento sísmico $\left(Q^{\prime}\right)$ es compleja, con un criterio simplista en esta evaluación se considera que numéricamente ambos parámetros son iguales. Con base en esta hipótesis y con fines de comparación, la fig 10 también muestra las demandas de resistencia especificadas por el Manual CFE93 para edificaciones a base de mampostería sin confinar $\left(Q^{\prime}=1\right)$ para la zona costera de Michoacán en suelo duro.

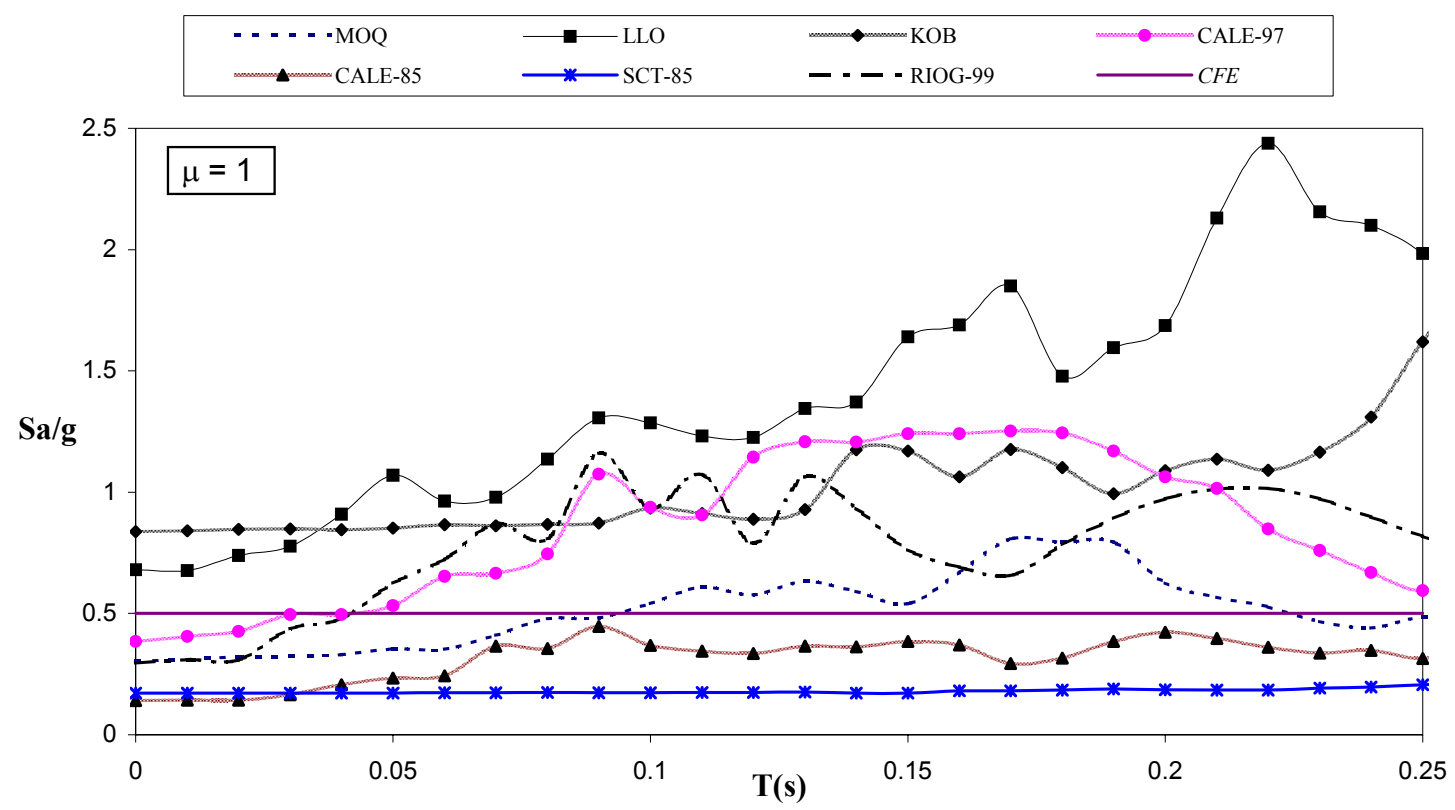

Figura 10. Espectros elásticos de aceleraciones para los registros analizados

Como se aprecia en la fig 10, las demandas de resistencia correspondientes al registro CALE-97 en algunos casos fueron más de dos veces las especificadas por el Manual CFE93. Esto sugiere que en estos casos las edificaciones de mampostería con comportamiento elástico, esto es edificaciones de adobe o de tabique sin confinar, que resistieron sin daños el terremoto de Caleta de Campos de 1997, debieron tener sobrerresistencias de más del doble de las especificadas por el referido Manual. Un comentario semejante es aplicable a edificaciones de estas características que resistieron el sismo de Oaxaca de 1999 ( fig 10). También es de interés mencionar que la mayor parte de registros llevan a demandas de resistencia generalmente mayores que las especificadas por el Manual CFE93. Además, de acuerdo con los resultados mostrados en la fig 10, las mayores demandas de resistencia en edificaciones de mampostería con comportamiento elástico corresponden al registro de LLolleo, obtenido en el sismo de Chile de 1985. Esto ayuda a explicar la gran destrucción de viviendas de adobe observadas en este sismo en la zona cercana a la estación donde se obtuvo este registro. Por el contrario, como se aprecia en la fig 10, las demandas de resistencia asociadas al registro SCT-85 en la ciudad de México son bastante menores que las demandas correspondientes a las de los otros registros analizados, lo que ayuda a explicar los pocos o nulos daños en edificaciones de mampostería sin confinar observados en la ciudad de México en el terremoto de 1985. 
Para el análisis de la respuesta sísmica de edificaciones de mampostería confinada en este trabajo se emplea la hipótesis de que a estas edificaciones se les puede asociar un valor global para $\mu$ igual a 2. Abundante información experimental (Meli, 1975; Ruiz, 1995; San Bartolomé, 2001) fundamenta esta hipótesis, en el sentido de que la mampostería confinada sometida a acciones laterales ha mostrado un comportamiento inelástico que no es despreciable, al cual en este trabajo de manera simplista se le asocia el valor de 2 para $\mu$. La fig 11 muestra los resultados de aplicar este criterio a los registros mencionados. Estos espectros se evaluaron con el programa USEE 2001 (2001), con el modelo de degradación de rigidez que emplea este programa. Además, se consideró un valor para $\xi$ igual a 0.05 . Estos resultados muestran que las demandas de resistencia asociadas a los registros de LLO y KOB son bastante mayores que las de los otros registros. Como se aprecia en la fig 12, el siguiente grupo de registros que le sigue en orden decreciente de demandas de resistencias, son CALE-97, MOQ y RIOG-99, cuyas demandas incluso superan a las demandas de resistencias especificadas por el Manual CFE93 para edificaciones a base de mampostería confinadas $\left(Q^{\prime}=2\right)$ para la zona costera de Michoacán en suelo duro. Por el contrario, los resultados de la fig 11 muestran que las demandas asociadas a los registros CALE-85 y SCT-85 son bastante menores que las especificadas por el Manual CFE93.

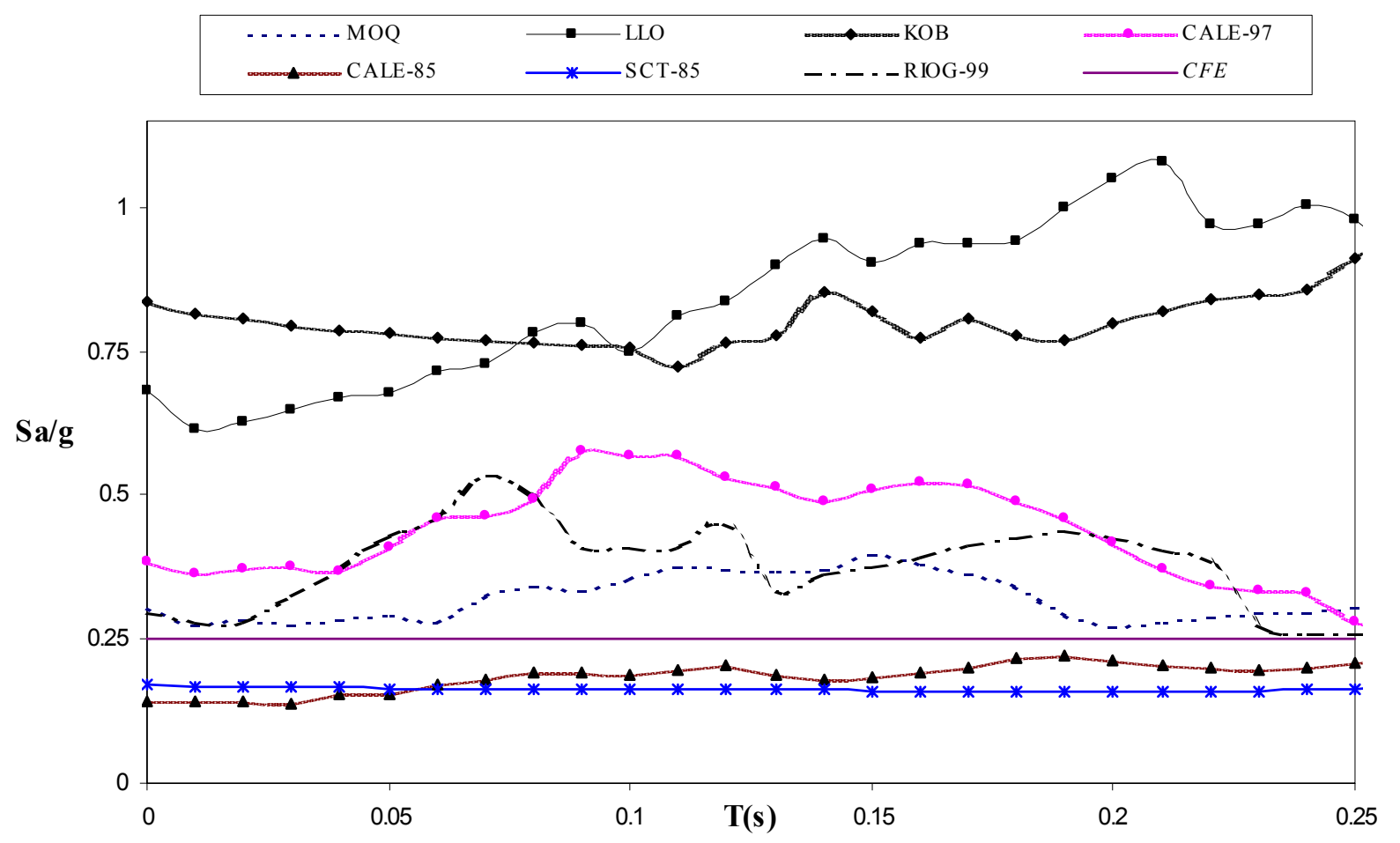

Figura 11. Espectros inelásticos $(\mu=2)$ de aceleraciones para los registros analizados

\section{Espectros de desplazamientos}

Tendencias semejantes a las arriba comentadas para demandas de resistencias se observa al analizar los espectros de desplazamientos, $S_{d}$, correspondientes a los registros analizados para 
estructuras con comportamiento elástico ( fig 12) ó con comportamiento inelástico asociado a un valor de $\mu$ igual a 2 ( fig 13). Este último caso se evaluó con el referido programa USEE 2001 (2001), con el modelo de degradación de rigidez mencionado. Estos resultados se emplean más adelante en un análisis comparativo de demanda y capacidad de deformación lateral de edificaciones de mampostería que son excitadas por los registros de aceleraciones mencionados.

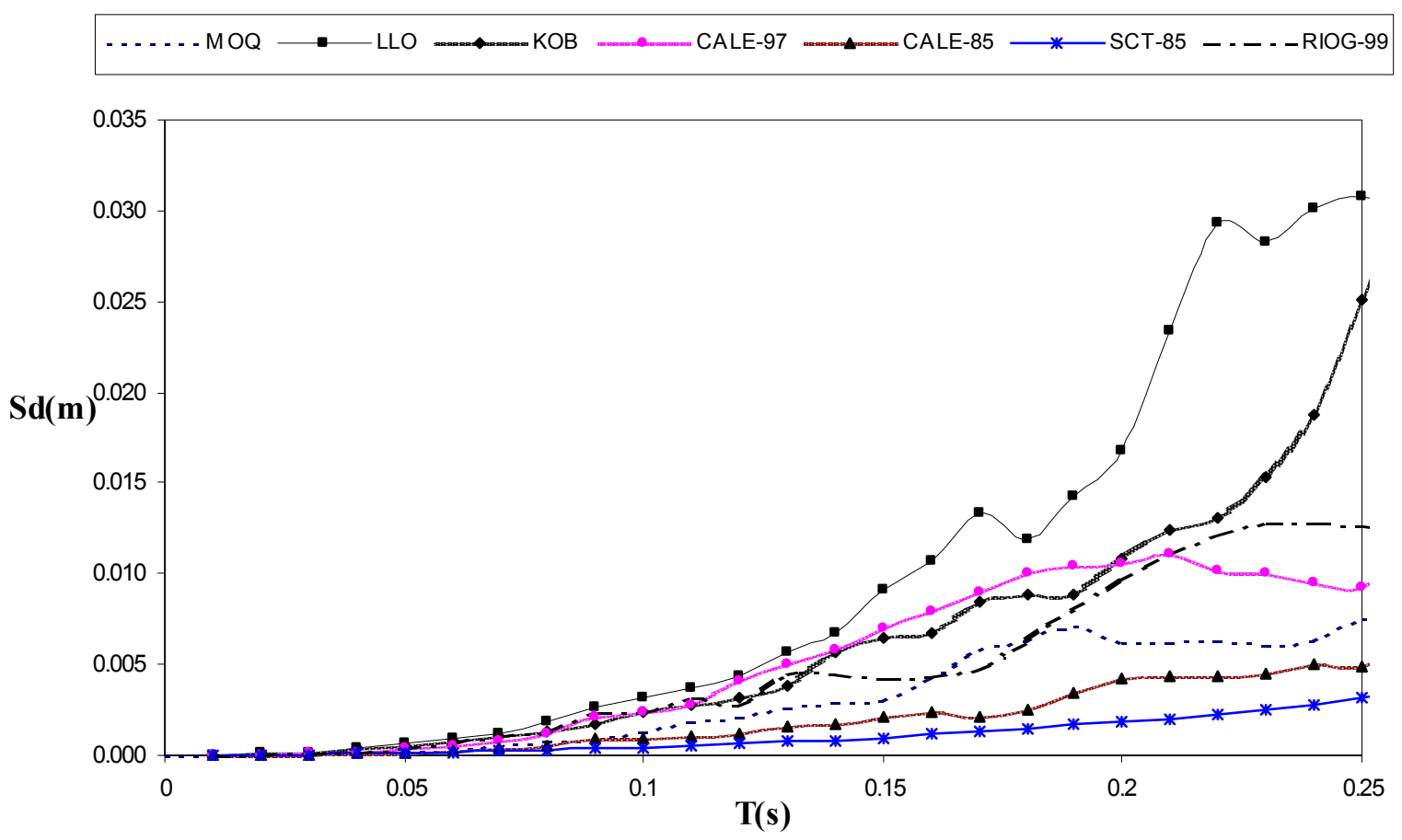

Figura 12. Espectros elásticos de desplazamientos para los registros analizados

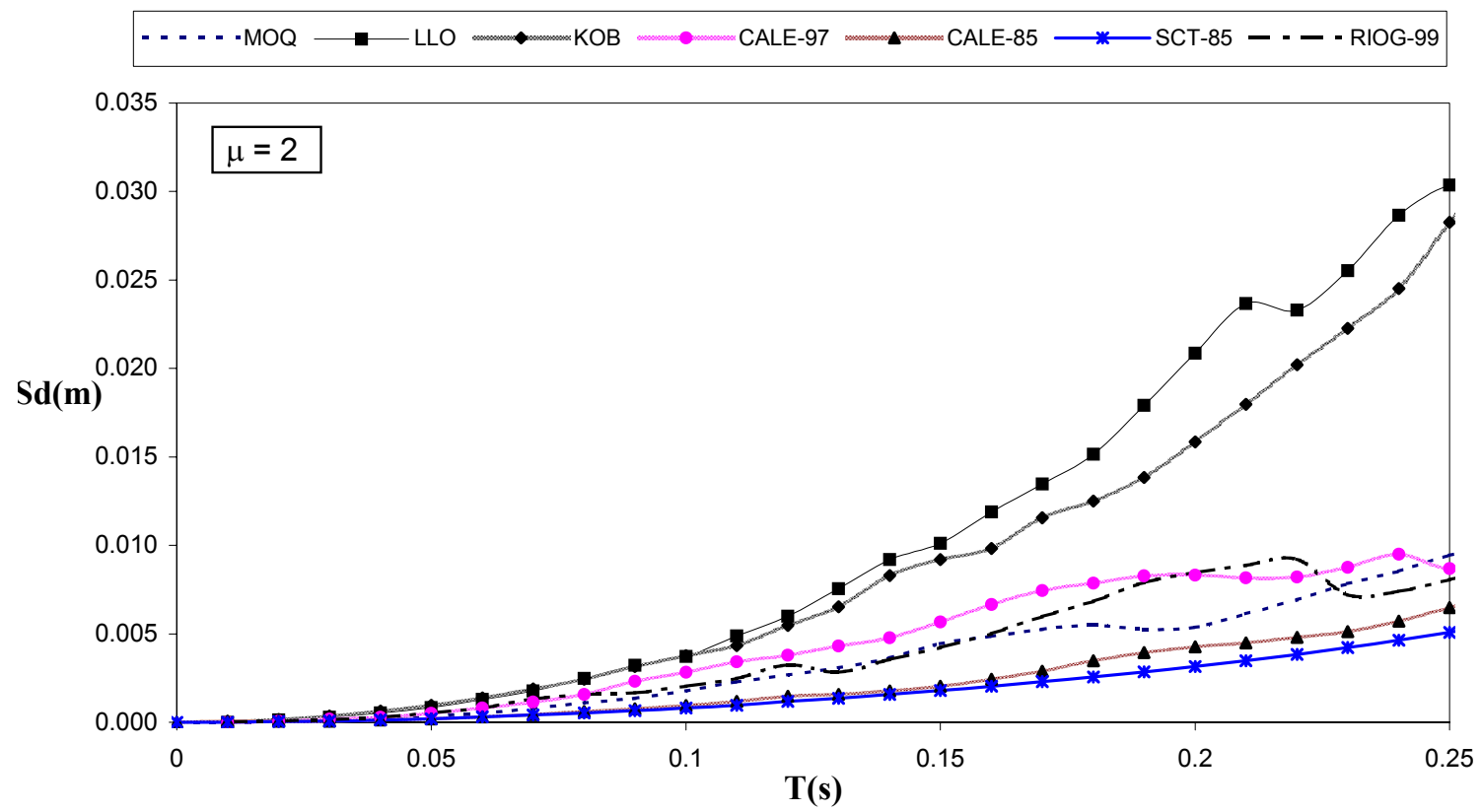

Figura 13. Espectros inelásticos $(\mu=2)$ de desplazamientos para los registros analizados 


\section{Análisis de demandas y capacidades resistentes de edificaciones de mampostería en función del índice de densidad de muros}

Un parámetro útil para evaluar el comportamiento sísmico de edificaciones de mampostería es el índice de densidad de muros, $p$, el cual se define como

$$
p=\frac{A_{m}}{A_{p}}
$$

donde $A_{m}$ es el área total de muros en la edificación en la dirección del análisis, y $A_{p}$ es el área de la planta.

Como se demuestra en lo que sigue, el parámetro $p$ se puede emplear como una medida de la resistencia requerida en una edificación de mampostería sometida a una excitación específica, representada por un registro de aceleraciones.

La fuerza cortante resistente global en edificaciones de mampostería, $V_{R}$, se puede evaluar como:

$$
V_{R}=v_{m} A_{m}
$$

donde $v_{m}$ es el esfuerzo cortante resistente en muros de mampostería.

Además, la fuerza cortante basal actuante, $V_{a c t}$, en una edificación de $n$ niveles, se puede evaluar de manera aproximada como:

$$
V_{a c t}=\frac{S a}{g} n \omega^{*} A_{p}
$$

donde $\omega^{*}$ es el peso por unidad de superficie en una planta típica de la edificación. Igualando (3) y (4), se obtiene:

$$
p=\frac{S a}{g} \frac{n \omega^{*}}{v_{m}}
$$

De acuerdo con el RCDF (1993), la expresión para $v_{m}$, sin considerar los factores respectivos de reducción de resistencia, $F_{R}$, está dada por:

$$
v_{m}=\left(0.5 v^{*}+0.3 \sigma\right) \leq 1.5 v^{*}
$$

donde $v^{*}$ es el esfuerzo cortante resistente de diseño en muros de mampostería.

El parámetro $\sigma$ es el esfuerzo vertical actuante sobre los muros de la edificación, y se puede evaluar como: 


$$
\sigma=\frac{W_{T}}{A_{m}}
$$

donde $W_{T}$ es el peso total del edificio Aún cuando los valores de $\sigma$ dependen de la densidad de muros y del tipo de sistema de piso, en este estudio se considera que una estimación razonable de este parámetro (en $\mathrm{kg} / \mathrm{cm}^{2}$ ) está dada por:

$$
\sigma=1.25 n
$$

La ec (8) se puede deducir de manera simplista considerando para edificaciones regulares una carga gravitacional por nivel de $800 \mathrm{~kg} / \mathrm{m}^{2}$, y una densidad de muros, $p$, de 0.064 .

Es posible relacionar $n$ y el periodo fundamental $T$, mediante la siguiente relación aproximada:

$$
T=\frac{n}{\lambda}
$$

donde el parámetro $\lambda$ depende de factores tales como tipo de estructura, excitación, densidad de muros, materiales, etc. Para este estudio se seleccionó el valor de 20 para $\lambda$, el cual resulta una aproximación razonable para construcciones típicas a base de muros de mampostería en suelo firme (Rodríguez et al., 1998).

Combinando las ecs (6), (8) y (9), y empleando los valores de $0.8 \mathrm{~kg} / \mathrm{cm}^{2}$ y $3.0 \mathrm{~kg} / \mathrm{cm}^{2}$ para $v^{*}$ en los casos de muros de adobe (Hernández et al., 1981) y de tabique confinado (RCDF, 1993), respectivamente, así como el valor de 20 para $\lambda$ se obtiene:

$$
\begin{array}{ll}
v_{m}=(0.4+7.5 T) \leq 1.2 & \text { para muros de adobe }(\mu=1) \\
v_{m}=(1.5+7.5 T) \leq 4.5 & \text { para muros de tabique confinados }\left(\mu_{-}=2\right)
\end{array}
$$

Es conveniente simplificar la ec (5) y expresarla en función de $T$, por lo que para este estudio el valor $\omega^{*}$ de se tomó igual a $0.08 \mathrm{~kg} / \mathrm{cm}^{2}$, lo cual proviene de estimar de manera conservadora el peso propio, la carga muerta y la carga viva en edificaciones de mampostería. Combinando las ecs (5), (9) y el valor propuesto de $0.08 \mathrm{~kg} / \mathrm{cm}^{2}$ para $\omega^{*}$ se obtiene:

$$
p=\frac{S a}{g} \frac{1.6 T}{v_{m}}
$$

Las figs 14 y 15 muestran resultados de aplicar las expresiones (10) a la (12) para evaluar los índices de densidad de muros requeridas en edificaciones de adobe y mampostería confinada, respectivamente, cuando la excitación corresponde a la de los registros de aceleraciones seleccionados. En estos resultado, así como en otros posteriores en este trabajo, en una segunda 
escala de abscisas se muestra el número de niveles de la edificación, lo cual se obtuvo empleando la ec (9) y el valor de 20 para $\lambda$. Se debe notar que en este estudio los máximos números de niveles que se consideran para edificaciones de adobe y de mampostería confinada son tres y cinco, respectivamente.

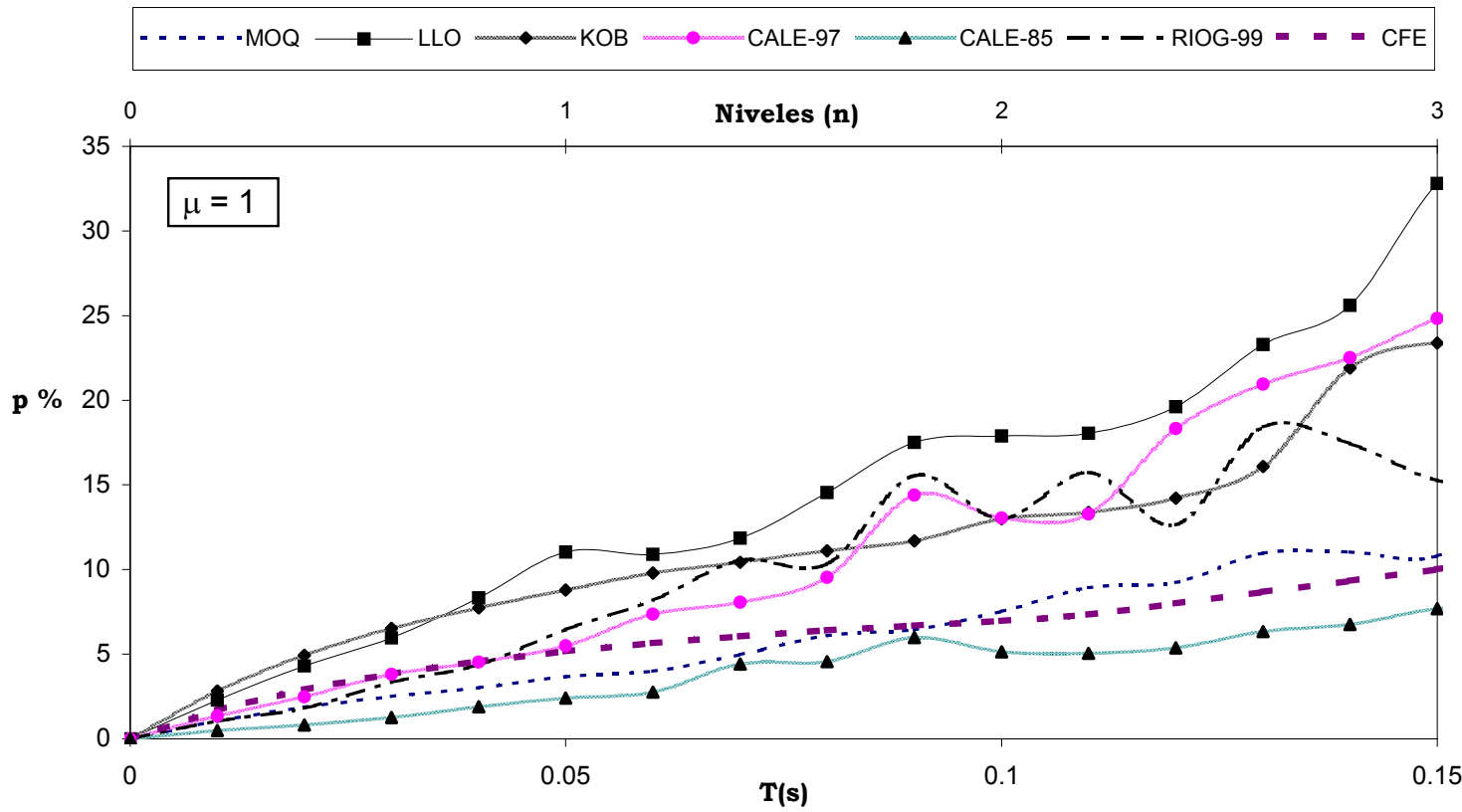

Figura 14. Demandas de densidad de muros $(p)$ considerando respuesta elástica para los registros analizados

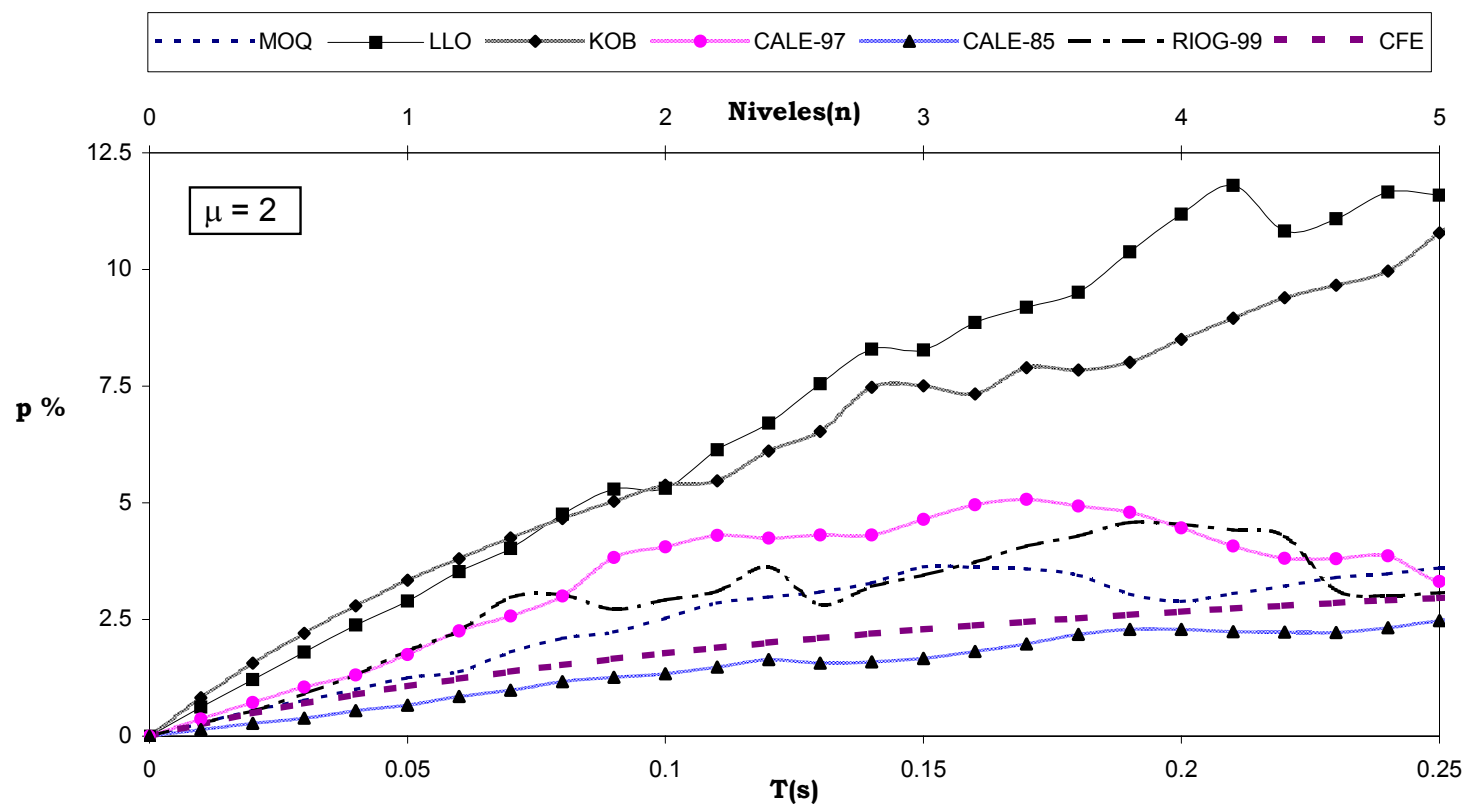

Figura 15. Demandas de densidad de muros $(p)$ considerando respuesta inelástica $(\mu=2)$ para los registros analizados 
Como se puede deducir de la inspección de la ec (12), para un mismo periodo y valor de $v_{m}, p$ es directamente proporcional a $S_{a} / g$, por lo que las características anteriormente comentadas para las demandas calculadas del parámetro $S_{a} / g$ correspondientes a los registros analizados serían también aplicables a $p$. Sin embargo, para el caso de una evaluación global los resultados mostrados en las figs 14 y 15 permiten comparar de manera sencilla las demandas requeridas de densidad de muros para resistir las acciones sísmicas representadas por los registros seleccionados y las densidades de muros que tendrían los casos en estudio. Por ejemplo, de acuerdo con los resultados de la fig 14, edificaciones de adobe de dos y tres niveles, con los esfuerzos cortantes resistentes supuestos para $v_{m}$, necesitarían tener una densidad de muros mayor que el $10 \%$ para resistir, con un comportamiento elástico, los movimientos sísmicos representados por los registros LLO, CALE-97, KOB y RIOG-99. Además, como se deduce de los resultados de la fig 15, el comportamiento inelástico (en este caso $\mu=2$ ) permitiría una reducción importante de la densidad requerida de muros con respecto al caso de respuesta elástica. En el caso de la mampostería confinada esta capacidad de deformación inelástica la da principalmente el confinamiento de castillos y dalas en los muros.

Se debe tener en cuenta que un modo de falla observado en viviendas de adobe sometidas a acciones sísmicas ha sido el de falla de muros fuera de su plano. Este modo de falla no se ha tomado en cuenta en esta investigación, ya que sólo se está considerando el tipo de falla de muro en su plano. Por lo anterior, dependiendo del comportamiento de muros fuera de su plano, podría regir el modo de falla asociado este comportamiento, aún cuando las edificaciones tengan las densidades de muro que se muestran en la fig 14.

\section{Análisis de demanda y capacidad de deformación lateral en edificaciones de mampostería}

Es importante poder incorporar resultados del análisis de la demanda y capacidad de deformación lateral de edificaciones de mampostería en procedimientos alternativos para la evaluación y diseño por sismo de este tipo de edificaciones, como el descrito en este trabajo. Con este objeto, en lo que sigue se hace este estudio de demanda y capacidad considerando los registros de aceleraciones seleccionados en este estudio.

Aún cuando el procedimiento que aquí se emplea es aplicable a casos específicos de edificaciones de mampostería, evaluando con mayor precisión algunas variables, este estudio se basa en una evaluación de tipo global, es decir aplicable a poblaciones típicas de edificaciones de mampostería.

En las figs 12 y 13 se han presentado los espectros de desplazamientos, $S_{d}$, para los registros seleccionados correspondientes a respuesta elástica $\left(\mu_{c}=1\right)$ e inelástica $\left(\mu_{c}=2\right)$. Sin embargo, rigurosamente su uso estaría limitado a estructuras de un grado de libertad y no representan los desplazamientos en edificaciones de varios niveles. Esta limitación se puede resolver aceptando algunas simplificaciones que pueden ser razonables si las estructuras son regulares. El criterio a emplear se basa en emplear una medida de la respuesta global de la estructura, esta medida es el desplazamiento lateral del último nivel de la edificación, $\delta$, en 
particular es de interés emplear el llamado desplazamiento relativo global, $D_{r m}$, el cual se define como:

$$
D_{r m}=\frac{\delta_{m}}{H}
$$

donde $\delta_{m}$ es el valor máximo de $\delta$, y $H$ es la altura del edificio. El parámetro $\delta_{m}$ se puede evaluar como:

$$
\delta_{m}=\gamma S_{d}
$$

donde $\gamma$ es igual a 1 para sistemas de un grado de libertad y para edificaciones regulares hasta de cinco niveles el valor 1.3 sería una estimación razonable (Rodríguez y Aristizábal, 1999).

Es de interés expresar $H$ en términos de $T$, lo que es posible hacer combinando (9), (13) y (14) (Rodríguez y Aristizábal, 1999), lo que lleva a

$$
D_{r m}=\frac{\gamma S_{d}}{\lambda h T}
$$

en la expresión anterior, $h$ es la altura de entrepiso.

Las figs 16 y 17 muestran resultados de aplicar la ec (15) para los registros seleccionados, para los casos de respuesta elástica $(\mu=1)$ e inelástica $(\mu=2)$, respectivamente. En ambos casos se consideró para $\xi$ el valor de 0.05 y en el caso inelástico se consideró un modelo de degradación de rigidez. Los valores de los parámetros $\gamma, \lambda$ y $h$ empleados para obtener estos resultados fueron $1.3,20$ y $250 \mathrm{~cm}$, respectivamente.

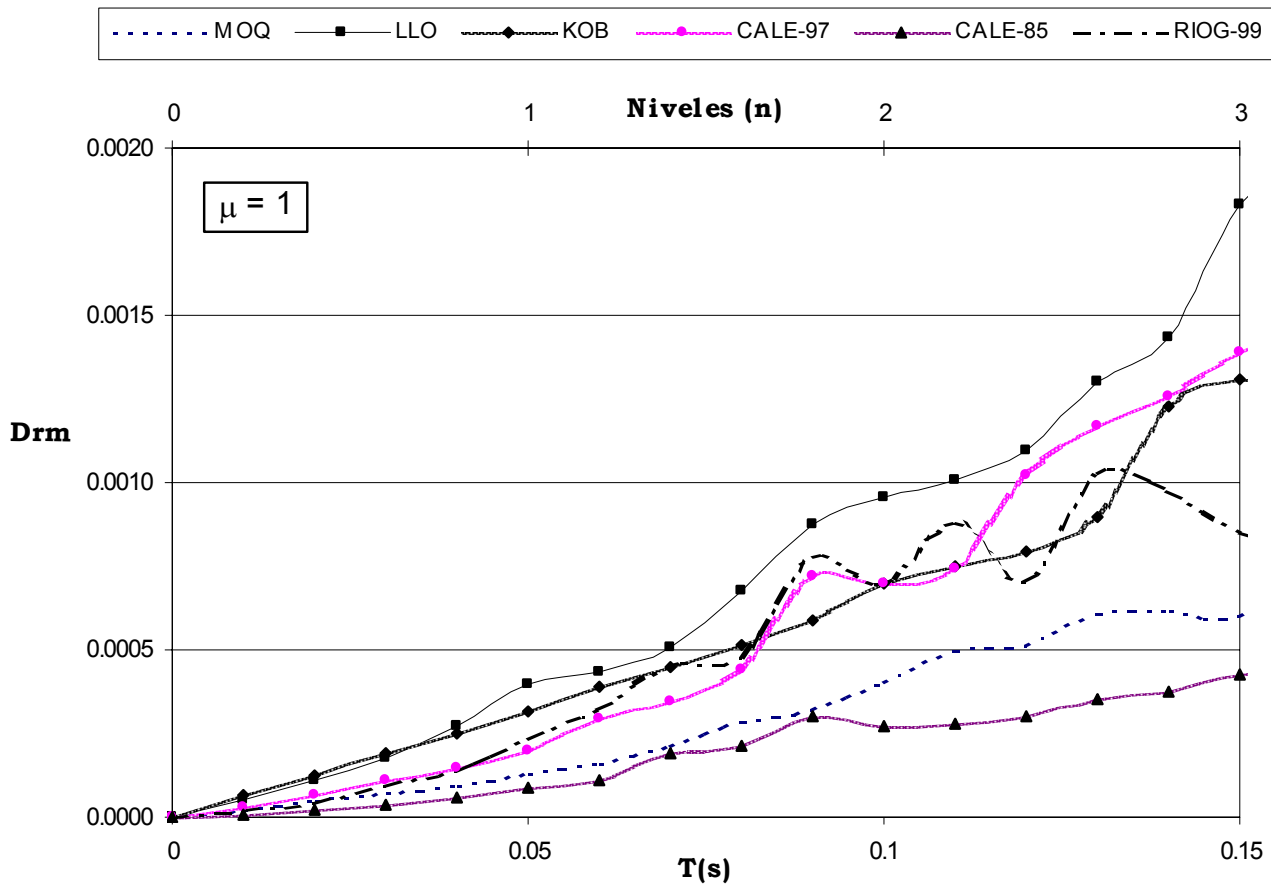

Figura 16. Demandas elásticas de deformación relativa global máxima 


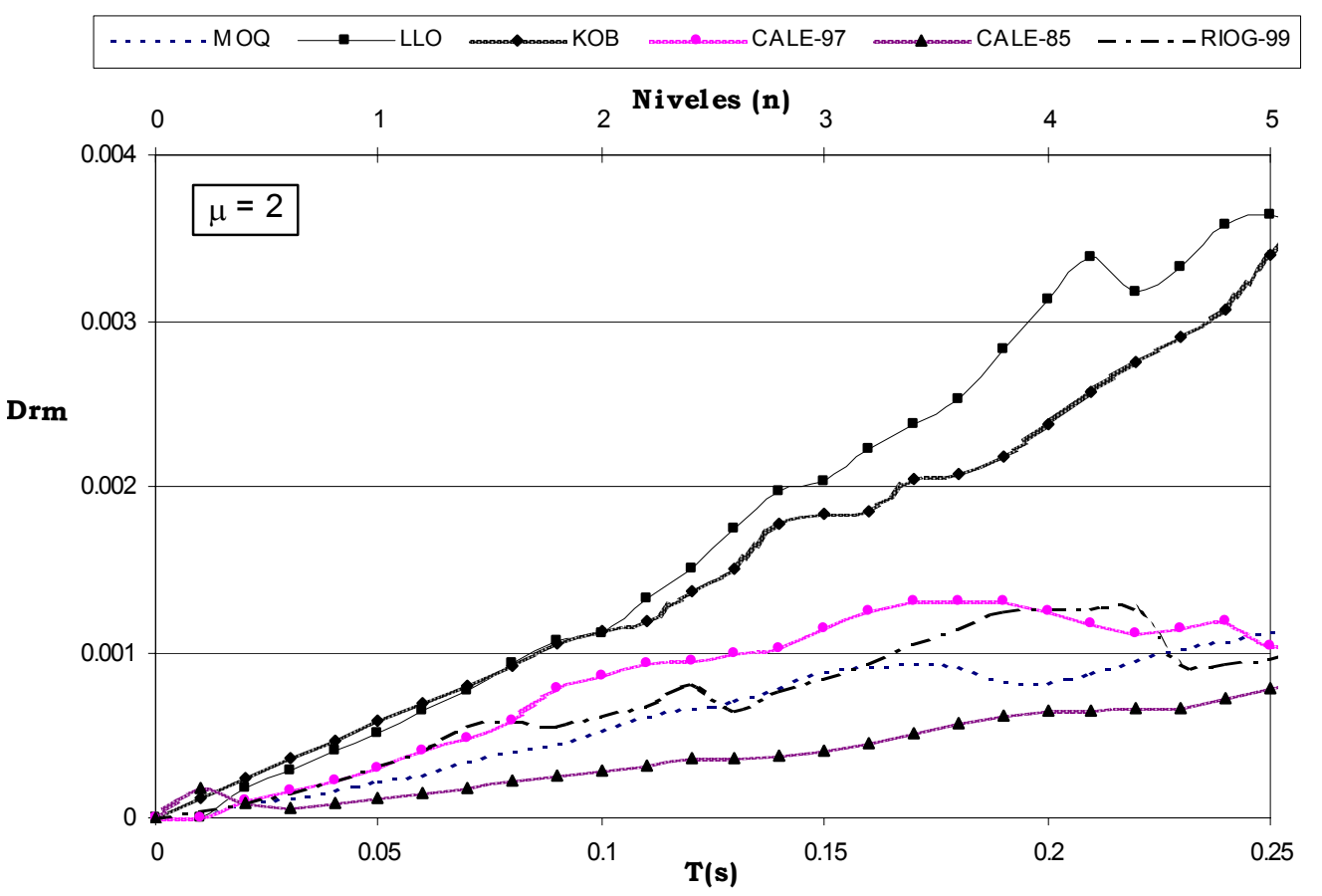

Figura 17. Demandas inelásticas $(\mu=2)$ de deformación relativa global máxima

Las capacidades de deformación se evalúan con el parámetro $D_{r d}$, el cual se define como el desplazamiento global relativo en la edificación asociado a daños sísmicos que se pueden considerar reparables (Rodríguez y Aristizábal, 1999). Aceptando de manera simplista que el comportamiento de muros de adobe es relevante en su plano, en el caso de edificaciones de este tipo, en este estudio se considera el intervalo entre 0.0005 y 0.001 como representativo para el parámetro $D_{r d}$ (Hernández et al., 1981; Rodríguez et al., 1998). Estos valores y los resultados de la fig 16 sugieren daños no reparables en edificaciones de adobe de dos y tres niveles sometidas a la mayor parte de las excitaciones seleccionadas para este estudio, particularmente para el registro de LLO.

Resultados de ensayes en laboratorio efectuados en México (Ruiz, 1995), empleando cargas laterales cíclicas reversibles en una edificación regular de dos niveles a base de muros confinados de tabique, muestran que para el valor de $D_{r m}$ igual a 0.0015 , la rigidez lateral global de la estructura se redujo al $50 \%$ de la rigidez inicial. También se observó que cuando $D_{r m}$ alcanzó el valor 0.003 , las grietas inclinadas de la mampostería penetraron en los castillos. Resultados comparables se encuentran en otras investigaciones (Gallegos, 1989; San Bartolomé, 2001). Estos resultados sugieren para $D_{r d}$ el intervalo entre 0.0015 y 0.0025 (Rodríguez et al., 1998). Estos valores y los resultados de la fig 17 sugieren que edificaciones regulares de mampostería confinada, de tres o más niveles, sometidas a las excitaciones de los registros LLO y $\mathrm{KOB}$, tendrían daños no reparables. De acuerdo con la fig 17, en este tipo de edificaciones las excitaciones correspondientes a los otros registros llevarían a daños menores, reparables.

Es útil relacionar $D_{r m}$ con la deformación relativa de entrepiso, $d_{r}$, más crítica en la edificación. Este parámetro es empleado por las recomendaciones del FEMA 273 anteriormente 
comentadas. En el caso de edificaciones de mampostería es posible encontrar una relación simple y útil entre $D_{r m}$ y $d_{r}$. En este trabajo esta relación se encuentra suponiendo que la respuesta sísmica se concentra en el primer nivel, es decir un modo de falla del tipo "piso débil" (Rodríguez et al., 2000), lo que ha sido observado en laboratorio en ensayes ante cargas cíclicas reversibles (Ruiz, 1995), así como en ensayes en mesa vibradora en dos especimenes a escala 1:5 representativos de edificaciones de mampostería confinada de tres niveles (Tomazevic y Klemenc, 1997). Una representación esquemática de este modo de falla se muestra en la fig 18. A partir del perfil de deformaciones mostrado en la fig 18 se puede deducir una relación entre la máxima deformación relativa de entrepiso, $d_{r m}$, y $D_{r m}$, la cual se puede expresar como:

$$
d_{r m}=n D_{r m}
$$

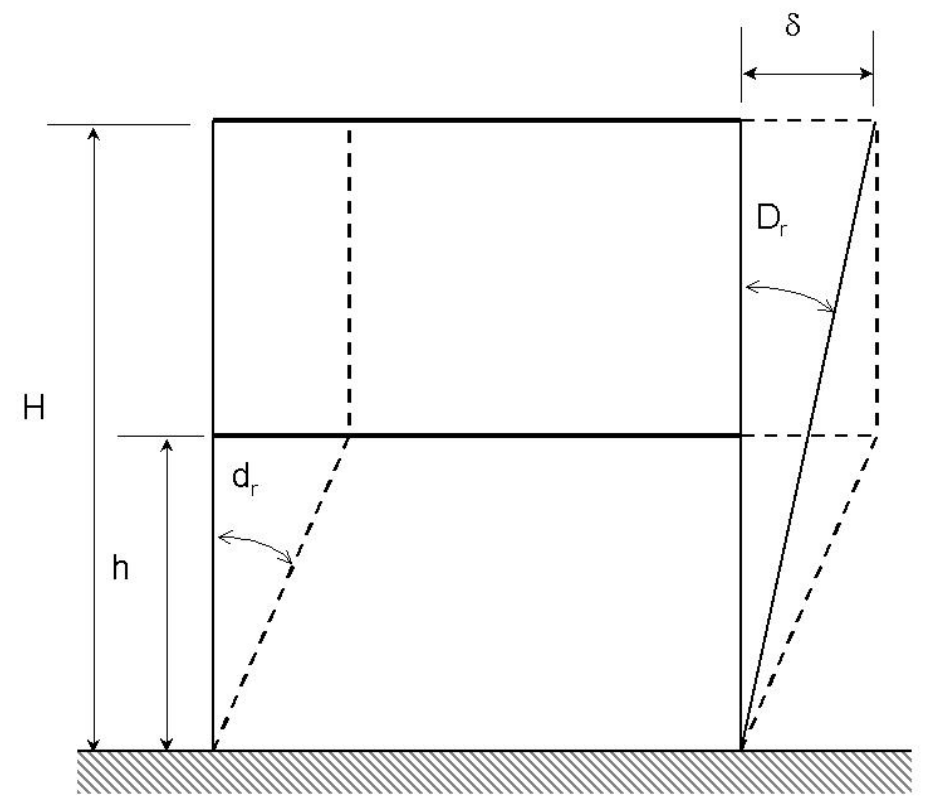

Figura 18. Modo de falla del tipo "piso débil" en una estructura de varios niveles

Las figs 19 y 20 muestran resultados del empleo de las ecs (15) y (16). En estos resultados los valores que intervienen de $D_{r m}$ son los mismos mostrados en las figs 16 y 17, respectivamente.

Aceptando en las edificaciones de muros de adobe tanto un comportamiento elástico $(\mu=1)$ como un modo de falla en el plano de los muros, los resultados de la fig 19 sugieren que en las edificaciones de dos y tres niveles con modo de falla del tipo "piso débil", la mayoría de los registros empleados, llevarían a demandas de deformaciones relativas de entrepiso mayores que 0.001 , es decir alcanzarían niveles de daños no reparables e incluso colapsos en algunos casos.

En los casos de edificaciones de mampostería confinada, los resultados de la fig 20 muestran que el modo de falla del tipo "piso débil" llevaría a valores de valores de deformaciones relativas de entrepiso mayores que 0.0025 en edificaciones de este tipo, de tres o más niveles, para la mayoría de registros de aceleraciones empleados, y por tanto estas edificaciones tendrían 
daños no reparables. En este tipo de edificaciones, los registros LLO y KOB llevan a superar el valor 0.0052 , que es el promedio experimental observado del parámetro $d_{2}$, el cual se ha definido en este estudio como el límite asociado al nivel de prevención de colapso.

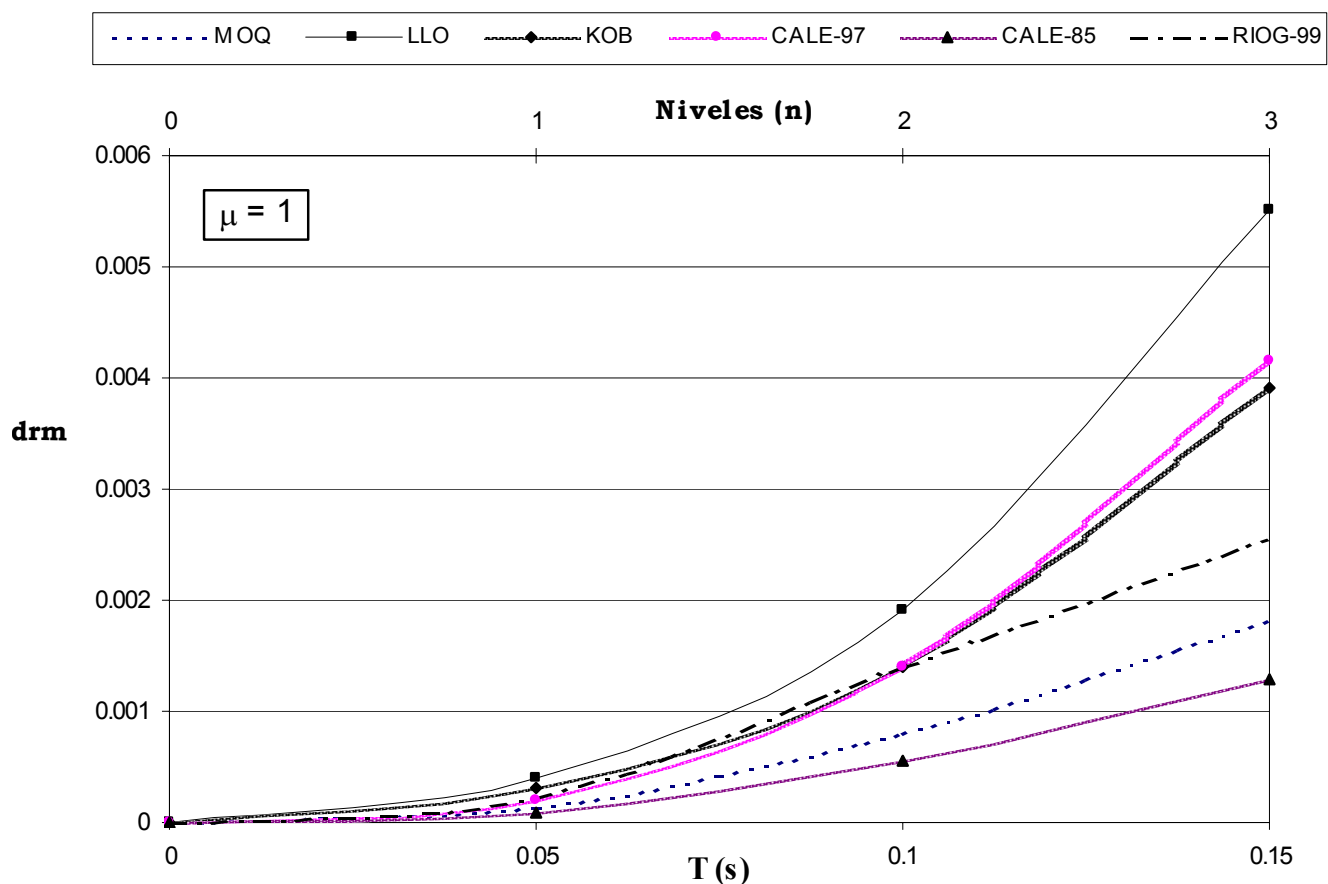

Figura 19. Demandas elásticas de deformaciones relativas de entrepiso en edificaciones de mampostería con modo de falla del tipo "piso débil"

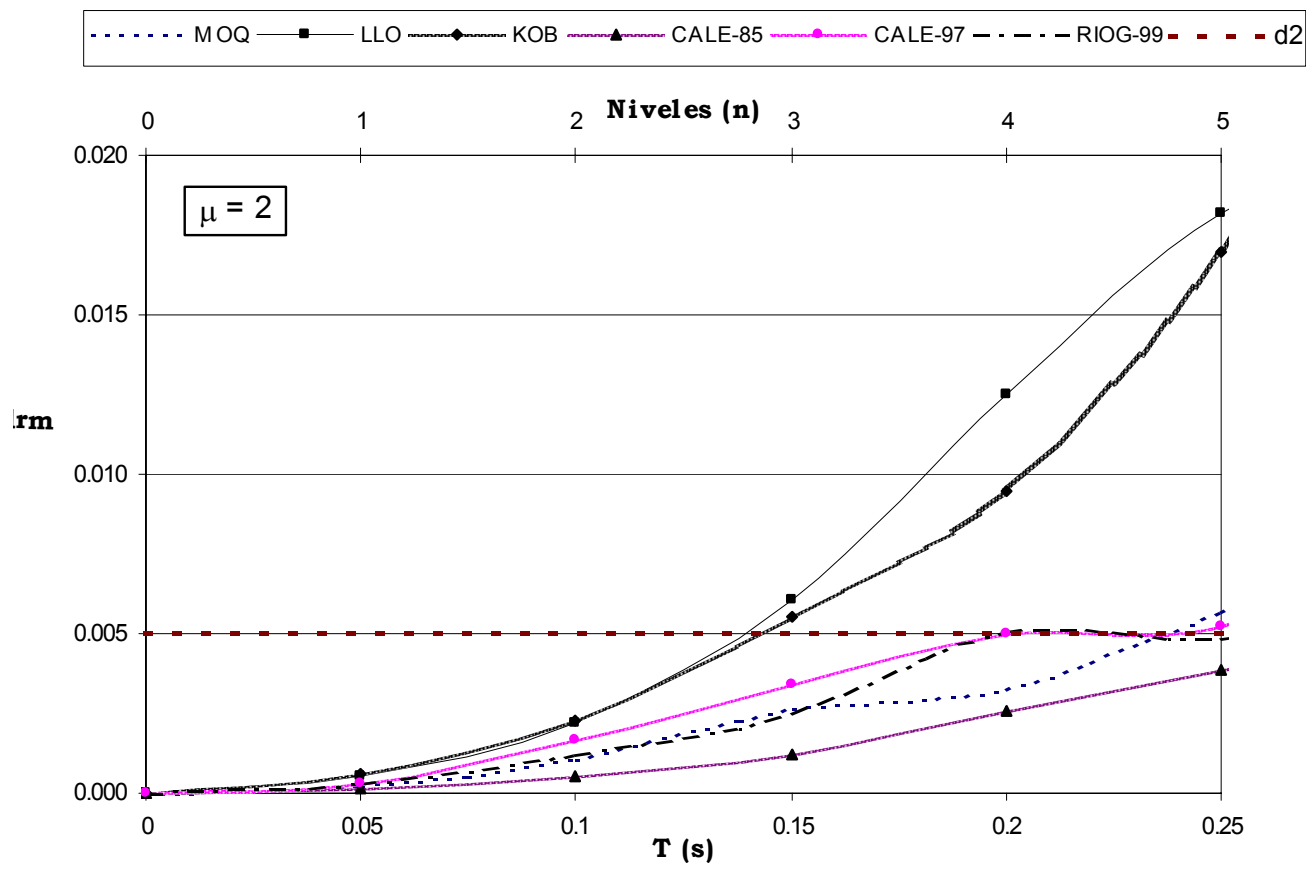

Figura 20. Demandas inelásticas $(\mu=2)$ de deformaciones relativas de entrepiso en edificaciones de mampostería con modo de falla del tipo "piso débil" 
Estos resultados indican la importancia de evitar en edificaciones de mampostería el modo de falla del tipo "piso débil", por las importantes demandas de desplazamientos asociadas a este modo de falla.

\section{EVALUACIÓN DE UNA MEDIDA DE DAÑO SÍSMICO EN EDIFICACIONES DE MAMPOSTERÍA CONFINADA}

En la literatura se ha propuesto el parámetro $I_{D}$, como medida del daño sísmico en edificaciones. Rodríguez (1994), y Rodríguez y Aristizábal (1999) han descrito en detalle esta medida y la han empleado para la evaluación del daño sísmico en edificaciones regulares de concreto reforzado. También el autor ha propuesto el empleo de esta medida de daño como herramienta para ser empleada en un procedimiento de diseño sísmico por desempeño (Rodríguez, 2002).

En este estudio el parámetro $I_{D}$ se emplea para la evaluación del daño en edificaciones de mampostería de tabique confinado. El parámetro $I_{D}$ no es aplicable al caso de edificaciones a base de muros de adobe, ya que para éstos se está considerando un comportamiento elástico. Este parámetro se define como (Rodríguez, 1994):

$$
I_{D}=\frac{\gamma^{2} E_{H}}{\left(2 \pi \lambda h D_{r d}\right)^{2}}
$$

En la expresión anterior $E_{H}$ es la energía histerética disipada al final del movimiento sísmico por una estructura de un grado de libertad con valores de $T$ y $\mu$, iguales, respectivamente, al periodo fundamental y relación máxima de ductilidad global de desplazamiento en la edificación.

La fig. 21 muestra espectros para el parámetro $I_{D}$, obtenidos con los registros seleccionados y con los mismos valores de los parámetros $\gamma, \lambda$ y $h$ empleados en la evaluación de $D_{r m}$. Además, para obtener estos espectros se empleó para $\xi$ el valor 0.05 , y se aceptó la hipótesis de que las edificaciones a base de muros de mampostería confinada pueden alcanzar una capacidad de ductilidad de desplazamiento, $\mu$, igual a 2. Para $D_{r d}$ se empleó el valor 0.0015 , el cual como se ha comentado, podría estar asociado a un nivel aceptable de daño sísmico en este tipo de edificaciones.

Los resultados de la fig 21 muestran que en las edificaciones de mampostería confinada el registro LLO llevaría a daños por sismo bastante mayores que los que provocarían los otros registros. Para edificaciones de mas de tres niveles, en orden decreciente de demandas de daños al registro LLO le sigue el registro KOB.

La fig 22 muestra a una escala mayor los mismos resultados de la fig 21 pero sin los resultados correspondientes a los registros LLO y KOB, mostrando los valores del parámetro $I_{D}$ correspondientes a los registros MOQ, CALE-97, CALE-85, SCT-85 y RIOG-99. Es de interés que en este grupo de registros el registro CALE-97 en un intervalo de periodos muestra valores de demandas de daños que son mayores que las de los otros registros. Además, los valores del 
parámetro $I_{D}$ para este registro del terremoto de Caleta de Campos de 1997 son bastante mayores que los correspondientes a los registros CALE-85 y SCT-85 obtenidos en el terremoto de Michoacán de 1985, lo que es congruente con la tendencia de daños observados en edificaciones de mampostería en ambos terremotos. Además, es de interés que de acuerdo con los resultados de las figs 21 y 22, los niveles de daños esperados en edificaciones de mampostería confinada para los registros MOQ y RIOG-99 (terremotos del Perú 2001 y Oaxaca 1999, respectivamente) son comparables.

INDICE DE DAÑO PARA MAMPOS TERIA

Modelo con degradacion de rigidez, $\mu=2, \lambda=20, \operatorname{Drd}=\mathbf{0 . 0 0 1 5}$

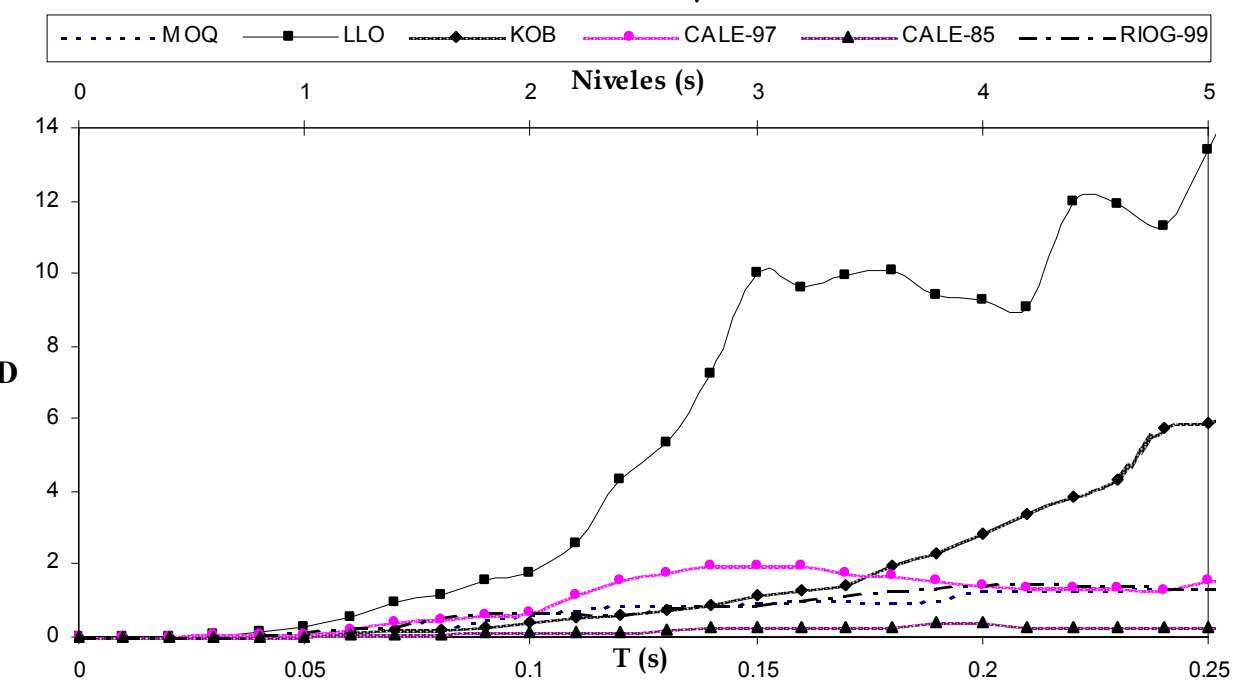

Figura 21. Parámetro de daño $I_{D}$ en edificaciones de mampostería para diversos registros de aceleraciones $(\mu=2 ; \xi=0.05)$

\section{INDICE DE DAÑO PARA MAMPOS TERIA}

Modelo con degradacion de rigidez, $\mu=2, \lambda=20$, Drd $=0.0015$

\begin{tabular}{|c|c|c|c|c|}
\hline$\ldots-M O Q$ & 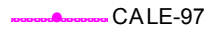 & CALE-85 & — SCT-85 & - - - - RIOG-99 \\
\hline 0 & 1 & Niveles (s) & 3 & 4 \\
\hline
\end{tabular}

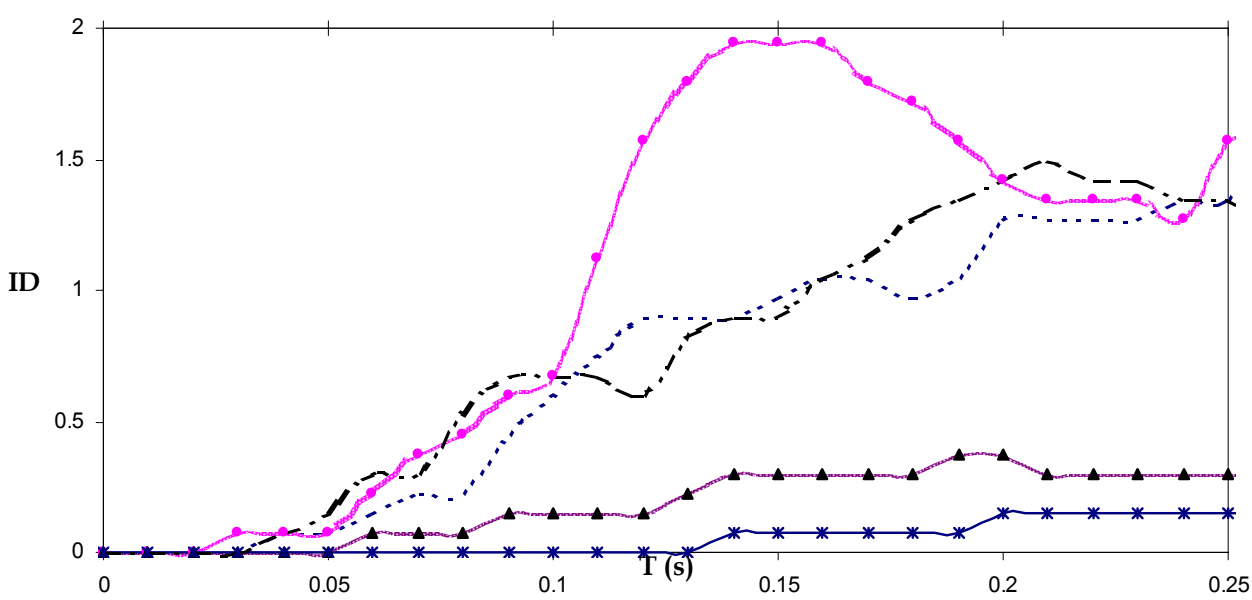

Figura 22. Parámetro de daño $I_{D}$ en edificaciones de mampostería para registros de aceleraciones de México (SCT-85, CALE-85, CALE-97 y RIOG-99) y Perú (MOQ), $(\mu=2 ; \xi=0.05$ ) 
La observación de la ec (17) muestra que el parámetro $I_{D}$ se puede relacionar con diferentes niveles de comportamiento definidos dentro de un criterio de diseño sísmico por desempeño. Para ello estos niveles de comportamiento estarían asociados a los valores seleccionados para $D_{r d}$ Para llegar a valores confiables del parámetro $I_{D}$ para empleo dentro del enfoque alternativo de diseño sísmico descrito en este trabajo sería necesario una calibración que considere registros de aceleraciones adicionales a los aquí empleados, así como considerar diferentes niveles de comportamiento sísmico esperado.

\section{CONCLUSIONES}

1. En este trabajo se hace énfasis en la evaluación de la demanda y capacidad de deformación de edificaciones de mampostería dentro de un enfoque alternativo de evaluación y diseño por sismo de este tipo de edificaciones. Con base en la evaluación de resultados de ensayes de especimenes de mampostería confinada se demuestra que la dispersión en resultados de la capacidad de deformación de estos especimenes es menor que la dispersión que se obtiene en la predicción de la capacidad resistente en ellos de acuerdo con reglamentos vigentes. Esta característica sugiere la conveniencia del empleo de un enfoque alternativo de diseño por sismo de edificaciones de mampostería, en el cual la evaluación de la demanda y capacidad de deformación de estas edificaciones debe ser un paso importante en el proceso de diseño.

2. En este estudio se emplea un parámetro de daño por sismo para la evaluación del comportamiento sísmico global de edificaciones de mampostería de tabique confinado. Esta medida emplea la energía histerética disipada por la estructura durante un terremoto, así como una medida de la capacidad de deformación de la estructura. Los resultados de la evaluación de esta medida de daño sísmico para los terremotos analizados son congruentes con los daños globales observados en estos terremotos. Estos resultados sugieren que este parámetro puede ser empleado dentro de criterios alternativos de diseño, tal como el llamado diseño sísmico por desempeño, para lo cual este parámetro debe calibrarse con un número mayor de sismos, así como empleando diferentes niveles de comportamiento sísmico esperado.

3. Como se deduce de los resultados de este estudio, así como de observaciones efectuadas por otros autores en sismos del tipo de intraplaca ocurridos en México y en otros países, los daños en edificaciones de mampostería causados por este tipo de sismos son mayores que los causados por sismos de igual magnitud pero del tipo de subducción de interplaca. Esta característica debiera tomarse en cuenta en los requisitos de diseño sísmico especificados por reglamentos para el caso de edificaciones de mampostería.

\section{AGRADECIMIENTOS}

Se agradece a L. P. Hoyos, becaria del II de la UNAM, quien colaboró en los cálculos numéricos que se presentan en este trabajo. Se agradece también los comentarios constructivos a este trabajo de los revisores anónimos. 


\section{REFERENCIAS}

Aguilar, G (1994), "Efecto del refuerzo horizontal en el comportamiento de mampostería confinada", Tesis de Licenciatura, Facultad de Ingeniería, UNAM.

Alcocer, S, R Meli y T Sánchez (1994), "Comportamiento ante cargas laterales de sistemas de muros de mampostería confinada con diferentes grados de acoplamiento a flexión", Centro Nacional de Prevención de Desastres (CENAPRED), cuaderno de investigación No. 17. México, D.F.

Anderson, J, R Quaas, S K Singh, D Almora, J Velasco, R Vázquez, C Pérez, M Ayala, G Castro y J Ylizaliturri (1997), "Accelerograms from the Guerrero, Mexico, strong motion array for the January 11, 1997 Caleta de Campos Earthquake $\left(\mathrm{M}_{\mathrm{w}}=7.1\right)$ ", Report GAA-19, UNR Seismological Laboratory e Instituto de Ingeniería, UNAM.

Arias, A (1970), "A measure of earthquake intensity", Seismic Design for Nuclear Power Plants (editado por R Hansen), Massachusetts Institute of Technology Press, Cambridge, USA, 438-483.

Astroza, M, O Díaz, M Pardo y S Rebolledo (2002), "Lecciones de un terremoto de subducción del tipo intraplaca. El terremoto de Punitaqui del 14 de octubre de 1997", Memorias de las Octavas Jornadas Chilenas de Sismología e Ingeniería Sísmica, Valparaíso, Chile.

Comisión Federal de Electricidad, CFE-93 (1993), "Manual de diseño de obras civiles. Diseño por sismo".

Flores, A (1993), "Preámbulo", en Ingeniería Sísmica. El caso del sismo del 3 de marzo de 1985, Instituto de Ingenieros de Chile, Chile.

FEMA-273 (1997), "NEHRP Guidelines for the Seismic Rehabilitation of Buildings", Federal Emergency Management Agency, Washington D.C.

Gallegos, H (1989), Albañilería Estructural, Pontificia Universidad Católica del Perú.

García, L E y L Yamin (1994), “Comportamiento sísmico de muros de mampostería confinada”, Facultad de Ingeniería, Universidad de los Andes, Bogota, Colombia.

Hernández, O, R Meli, M Padilla y E Valencia (1981), "Refuerzo de la vivienda económica en zonas sísmicas. Estudios experimentales", Publicación 441 de la Serie Azul del Instituto de Ingeniería de la UNAM.

Herrera, E (1992), "Efectos de la carga vertical en el comportamiento de muros de albañilería reforzada sometida a carga lateral alternada", Tesis Profesional, Facultad de Ciencias Físicas y Matemáticas, Universidad de Chile, Chile.

Lermo, J, F Lázares y J Cuenca (2002), "El terremoto de Arequipa, Perú del 23 de junio del 2001 $\left(\mathrm{M}_{\mathrm{w}}=8.2\right)$, efectos de sitio en las ciudades de Arequipa, Camaná, Moquegua, Ilo y Tacan y su relación con los daños en edificaciones", Memorias de las Octavas Jornadas Chilenas de Sismología e Ingeniería Sísmica, Valparaíso, Chile.

Magenes, G y G M Calvi (1997), "In-plane seismic response of brick masonry walls", Earthquake Engineering \& Structural Dynamics Journal, Vol. 26, pp. 1091-1112.

Meli, R (1975), "Comportamiento sísmico de muros de mampostería”, Informe 352, Instituto de Ingeniería, UNAM, México, D.F. 
Meli, R y G Salgado (1969), “Comportamiento de muros de mampostería sujetos a carga lateral”, Informe 237, Instituto de Ingeniería, UNAM, México, D.F.

Muguruma, H, M Nisiyama y F Watanabe (1995), "Lessons learned from the Kobe EarthquakeA Japanese perspective", PCI Journal, pp. 28-42, julio-agosto.

Muñoz, A y B Tinman (2001), "El sismo de Arequipa del 2001 y la vulnerabilidad de las edificaciones peruanas", Reporte DI-SIC-2001-07, Departamento de Ingeniería Civil, Pontificia Universidad Católica del Perú.

New Zealand Reconnaissance Team (1988), "The September 1985 Mexico Earthquakes. Final Report of the New Zealand Reconnaissance Team", Bulletin of the New Zealand National Society for Earthquake Engineering, Vol. 21, No. 1, pp. 3-96.

Reglamento de Construcciones para el Distrito Federal RCDF-93 (1993), Gaceta Oficial del Departamento del D. F.

Rodriguez, M (1994), "A measure of the capacity of earthquake ground motions to damage structures", Earthquake Engineering and Structural Dynamics, Vol. 23, No. 6, pp. 627-643.

Rodríguez, M, P Alarcón y R Machicao (1998), "Evaluación del comportamiento sísmico de edificaciones de mampostería en los sismos de Michoacán de 1985 y 1997", Revista de Ingeniería Sísmica, SMIS, No. 59, pp. 37-58.

Rodríguez, M y J C Aristizábal (1999), "An evaluation of a seismic damage parameter", Earthquake Engineering and Structural Dynamics, Vol. 28, pp. 463-477.

Rodriguez, M y V Rodriguez (2000), "Performance-based earthquake-resistant design of confined masonry walls", Memorias, 12th World Conference on Earthquake Engineering, Auckland, Nueva Zelanda.

Rodríguez, M (2002), "Diseño por desempeño de estructuras de concreto reforzado", Memorias, VII Simposio Nacional de Ingeniería Sísmica, organizado por SMIS, Cuernavaca, Morelos, noviembre, CD-ROM.

Rosenblueth, E y S E Ruiz, editores (1989), “The Mexico Earthquake of September 19, 1985”, Earthquake Spectra, Vol. 5, No. 1.

Ruiz, J (1995), "Reparación y refuerzos de una estructura tridimensional de mampostería", Tesis de Maestría, División de Estudios de Posgrado, Facultad de Ingeniería, UNAM.

San Bartolomé, A (2001), Construcciones de albañilería-comportamiento sísmico y diseño estructural, Pontificia Universidad Católica del Perú, fondo editorial, tercera edición.

Saragoni R, A Sáez y A Holmberg (1993), "Análisis de los acelerogramas del sismo de marzo de 1985", en "Ingeniería Sísmica. El caso del sismo del 3 de marzo de 1985”. Instituto de Ingenieros de Chile, Chile.

Singh, S K, M Ordaz, L Alcántara, N Shapiro, V Kostoglodov, J Pacheco, S Alcocer, C Gutiérrez, R Quaas, T Mikumo y E Ovando(2000), "The Oaxaca Earthquake of 30 September $1999\left(\mathrm{M}_{\mathrm{w}}=7.5\right)$ : a normal-faulting event in the subducted Cocos plate", Seismological Research Letters, Vol 71, No. 1, pp. 67-77. 
Tomazevic, M e I Klemenc (1997), "Verification of seismic resistance of confined masonry buildings", Earthquake Engineering and Structural Dynamics, Vol. 26, pp. 1073-1088.

USEE-2001 (2001), "Utility Software for Earthquake Engineering", desarrollado por el MidAmerican Earthquake Center, University of Illinois, USA.

Zegarra, L, A San Bartolomé y D Quiun (2001), “Comportamiento ante el terremoto del 23-062001 de las viviendas de adobe reforzadas en Moquegua, Tacna y Arica”, Reporte DI-SIC-200108, Departamento de Ingeniería Civil, Pontificia Universidad Católica del Perú. 